\title{
The Cauchy Problem for Nonlinear Klein-Gordon Equations in the Sobolev Spaces
}

\author{
By \\ Makoto NaKamurA* and Tohru OzaWA**
}

\begin{abstract}
The local and global well-posedness for the Cauchy problem for a class of nonlinear Klein-Gordon equations is studied in the Sobolev space $H^{s}=H^{s}\left(\mathbb{R}^{n}\right)$ with $s \geq n / 2$. The global well-posedness of the problem is proved under the following assumptions: (1) Concerning the nonlinearity $f, f(u)$ behaves as a power $u^{1+4 / n}$ near zero. At infinity $f(u)$ has an exponential growth rate such as $\exp \left(\kappa|u|^{\nu}\right)$ with $\kappa>0$ and $0<\nu \leq 2$ if $s=n / 2$, and has an arbitrary growth rate if $s>n / 2$. (2) Concerning the Cauchy data $(\phi, \psi) \in \mathcal{H}^{s} \equiv H^{s} \oplus H^{s-1},\left\|(\phi, \psi) ; \mathcal{H}^{1 / 2}\right\|$ is relatively small with respect to $\left\|(\phi, \psi) ; \dot{\mathcal{H}}^{s^{*}}\right\|$, where $s^{*}$ is a number with $s^{*}=n / 2$ if $s=n / 2$, $n / 2<s^{*} \leq s$ if $s>n / 2$, and the smallness of $\left\|(\phi, \psi) ; \dot{\mathcal{H}}^{n / 2}\right\|$ is also needed when $s=n / 2$ and $\nu=2$.
\end{abstract}

\section{$\S 1 . \quad$ Introduction}

We consider the local and global well-posedness in the Sobolev space $H^{s}=$ $H^{s}\left(\mathbb{R}^{n}\right)=(1-\Delta)^{-s / 2} L^{2}\left(\mathbb{R}^{n}\right)$ of fractional order $s$ with $s \geq n / 2$ for the Cauchy problem for nonlinear Klein-Gordon equations of the form

$$
\partial_{t}^{2} u-\Delta u+u=f(u)
$$

in space-time $I \times \mathbb{R}^{n}$, where $I=[-T, T]$ with $T>0$ for local solutions and $I=\mathbb{R}$ for global solutions. Here $u$ is a complex-valued function of $(t, x) \in I \times \mathbb{R}^{n}$, $\partial_{t}^{2}=\partial^{2} / \partial t^{2}, \Delta$ is the Laplacian in $\mathbb{R}^{n}$, and $f(u)$ is a nonlinear interaction given by a complex-valued function $f$ on $\mathbb{C}$.

Communicated by T. Kawai, August 21, 2000.

2000 Mathematics Subject Classification(s): 35L70.

* Graduate School of Information Sciences(GSIS), Tohoku University, Sendai 980-8577, Japan.

** Department of Mathematics, Hokkaido University, Sapporo 060-0810, Japan. 
There is a large literature on the Cauchy problem for (1.1) and on the asymptotic behavior in time of global solutions. We only refer to $[7,11,19]$ at the level of $H^{1}$ and $[2,12]$ at the level of $H^{2}$ for global solutions of (1.1) with some structural assumption on $f(u)$, and to $[20,21,23,27,28]$ for global solutions of (1.1) at the level of $H^{1}$ with small data setting. In [29, 30], B. Wang developed the $H^{s}$-theory for (1.1) for $1 / 2 \leq s<n / 2$.

To state the main result precisely we introduce the following notation. $\mathcal{H}^{s}$ denotes the Hilbert space $H^{s} \oplus H^{s-1}$ with norm

$$
\left\|(\phi, \psi) ; \mathcal{H}^{s}\right\| \equiv\left\{\left\|\phi ; H^{s}\right\|^{2}+\left\|\psi ; H^{s-1}\right\|^{2}\right\}^{1 / 2} .
$$

For any $r$ with $1 \leq r \leq \infty, L^{r}=L^{r}\left(\mathbb{R}^{n}\right)$ denotes the Lebesgue space on $\mathbb{R}^{n}$. For any $s \in \mathbb{R}$ and any $r$ with $1<r<\infty, H_{r}^{s}=(1-\Delta)^{-s / 2} L^{r}$ denotes the Sobolev space defined in terms of Bessel potentials. For any $s \in \mathbb{R}$ and any $r$ with $1<r<\infty, \dot{H}_{r}^{s}=(-\Delta)^{-s / 2} L^{r}$ denotes the homogeneous Sobolev space defined in terms of Riesz potentials. To introduce the Besov space and the homogeneous Besov space, let $\phi_{0}$ be a nonnegative function on $\mathbb{R}^{n}$ with

$$
\operatorname{supp} \phi_{0} \subset\left\{\xi \in \mathbb{R}^{n} ; 1 / 2 \leq|\xi| \leq 2\right\}
$$

such that $\left\{\phi_{0}\left(2^{-j} \cdot\right)\right\}_{j=-\infty}^{\infty}$ forms the Littlewood-Paley dyadic decomposition on $\mathbb{R}^{n} \backslash\{0\}$. Let $\left\{\psi_{j}\right\}_{-\infty}^{\infty}$ and $\tilde{\psi}$ be functions defined by

$$
\mathcal{F} \psi_{j}(\xi) \equiv \phi_{0}\left(2^{-j} \xi\right), \quad \mathcal{F} \tilde{\psi}(\xi) \equiv 1-\sum_{j=1}^{\infty} \phi_{0}\left(2^{-j} \xi\right),
$$

where $\mathcal{F}$ and $\mathcal{F}^{-1}$ denote Fourier transform and its inverse, respectively. For any $s \in \mathbb{R}$ and any $r, m$ with $1 \leq r, m \leq \infty$, we define

$$
\begin{aligned}
& \left\|u ; B_{r, m}^{s}\right\| \equiv\left\{\left\|\tilde{\psi} * u ; L^{r}\right\|^{m}+\sum_{j=1}^{\infty}\left(2^{s j}\left\|\psi_{j} * u ; L^{r}\right\|\right)^{m}\right\}^{1 / m}, \\
& \left\|u ; \dot{B}_{r, m}^{s}\right\| \equiv\left\{\sum_{j=-\infty}^{\infty}\left(2^{s j}\left\|\psi_{j} * u ; L^{r}\right\|\right)^{m}\right\}^{1 / m},
\end{aligned}
$$

where $*$ denotes the convolution in $\mathbb{R}^{n}$. Then the Besov space $B_{r, m}^{s}$ and the homogeneous Besov space $\dot{B}_{r, m}^{s}$ are defined by

$$
\begin{aligned}
& B_{r, m}^{s} \equiv\left\{u \in \mathcal{S}^{\prime}\left(\mathbb{R}^{n}\right) ;\left\|u ; B_{r, m}^{s}\right\|<\infty\right\}, \\
& \dot{B}_{r, m}^{s} \equiv\left\{u \in \mathcal{S}^{\prime}\left(\mathbb{R}^{n}\right) / \mathcal{P}\left(\mathbb{R}^{n}\right) ;\left\|u ; \dot{B}_{r, m}^{s}\right\|<\infty\right\},
\end{aligned}
$$


where $\mathcal{S}^{\prime}\left(\mathbb{R}^{n}\right)$ and $\mathcal{P}\left(\mathbb{R}^{n}\right)$ denote the sets of tempered distributions and of the polynomials on $\mathbb{R}^{n}$, respectively. We refer to $[1,6,26]$ for general information on Besov and Triebel-Lizorkin spaces and their homogeneous counterparts. For simplicity we make abbreviations such as $H^{s}=H_{2}^{s}, \dot{H}^{s}=\dot{H}_{2}^{s}, B_{r}^{s}=B_{r, 2}^{s}, \dot{B}_{r}^{s}=$ $\dot{B}_{r, 2}^{s}$. For any Banach spaces $X$ and $Y$ having a common dense subspace, we put

$$
\|a ; X \cap Y\| \equiv \max \{\|a ; X\|,\|a ; Y\|\}
$$

for any $a \in X \cap Y$. For any interval $I \subset \mathbb{R}$ and any Banach space $X$ we denote by $C(I ; X)$ the space of strongly continuous functions from $I$ to $X$, by $L^{q}(I ; X)$ the space of measurable functions $u$ from $I$ to $X$ such that $\|u(\cdot) ; X\| \in L^{q}(I)$, and by $C_{b}(I ; X)$ the space $\left(C \cap L^{\infty}\right)(I ; X)$. To describe the free propagator which solves the free Klein-Gordon equations, we define the operators $U(t) \equiv \exp (i t \omega)$, $K(t) \equiv \sin (t \omega) / \omega, \dot{K}(t) \equiv \cos (t \omega)$, where $\omega \equiv(1-\Delta)^{1 / 2}$. For any $r$ with $1 \leq r \leq \infty, r^{\prime}$ is the exponent dual to $r$ defined by $1 / r+1 / r^{\prime}=1$.

The Cauchy problem for the equation (1.1) with given data $(\phi, \psi)$ will be treated in the form of the integral equation

$$
u(t)=\Phi(u)(t) \equiv \dot{K}(t) \phi+K(t) \psi+\int_{0}^{t} K(t-\tau) f(u(\tau)) d \tau .
$$

To describe the nonlinear interaction $f$ with large growth at infinity as well as with a vanishing behavior as a power $p$ at zero, we introduce the following assumption $N(s, \mu, p)$ with $n / 2 \leq s, 0 \leq \mu \leq s$ and $1 \leq p<\infty$.

$N(s, \mu, p): f \in C^{[\mu]}(\mathbb{C} ; \mathbb{C})$ and for all $k$ with $0 \leq k \leq[\mu], f^{(k)}$ satisfies the estimates $\left|f^{(k)}(z)\right| \leq|z|^{(p-k)+} M(|z|)$ and

$$
\begin{aligned}
\mid f^{([\mu])}\left(z_{1}\right) & -f^{([\mu])}\left(z_{2}\right) \mid \\
& \leq \begin{cases}\left|z_{1}-z_{2}\right|^{p-[\mu]} M\left(\left|z_{1}\right| \vee\left|z_{2}\right|\right) & \text { if } \mu<p<[\mu]+1, \\
\left|z_{1}-z_{2}\right|\left(\left|z_{1}\right| \vee\left|z_{2}\right|\right)^{(p-[\mu]-1)+} M\left(\left|z_{1}\right| \vee\left|z_{2}\right|\right) & \text { otherwise }\end{cases}
\end{aligned}
$$

for all $z, z_{1}, z_{2} \in \mathbb{C}$, where $M(x) \equiv C \exp \left(\kappa|x|^{\nu}\right)$ with constants $\kappa>0$, $0<\nu \leq 2, C>0$ if $s=n / 2$, and $M$ is a nonnegative, nondecreasing function on $\mathbb{R}_{+}$if $s>n / 2$.

Here $f^{(k)}$ denotes any of the $k$-th order derivatives of $f$ with respect to $z$ and $\bar{z}$ and $\left|f^{(k)}\right|$ denotes the maximum of the moduli of those derivatives. We note that for any $\mu_{1}, \mu_{2}$ and $s$ with $0 \leq \mu_{1}<\mu_{2} \leq s<\infty$ and any $p$ with $1 \leq p<\infty, N\left(s, \mu_{2}, p\right)$ implies $N\left(s, \mu_{1}, p\right)$. For $a, b \in \mathbb{R}$ we denote by $a \vee b$ and 
$a \wedge b$ the maximum and minimum of $a$ and $b$, respectively, by $a_{+}$the maximum of $a$ and 0 , and by $[a]$ the greatest integer that is less than or equal to $a$. For any $0 \leq s_{0} \leq s^{*} \leq s$ we define the set of data by

$$
A^{s}\left(\gamma_{s_{0}}, \gamma_{s^{*}}\right) \equiv\left\{(\phi, \psi) \in \mathcal{H}^{s} ;\left\|(\phi, \psi) ; \mathcal{H}^{s_{0}}\right\| \leq \gamma_{s_{0}}, \quad\left\|(\phi, \psi) ; \mathcal{H}^{s^{*}}\right\| \leq \gamma_{s^{*}}\right\} .
$$

We are now in a position to state our main theorem in this paper.

Theorem 1.1. Let $n \geq 1, s \geq n / 2,1 \leq p<\infty$. Let $s^{*}$ be a number with $s^{*}=n / 2$ if $s=n / 2, n / 2<s^{*} \leq s$ if $s>n / 2$. Let $f$ satisfy $N\left(s,(s-1)_{+}, p\right)$. Let $\left\|(\phi, \psi) ; \mathcal{H}^{n / 2}\right\|$ be sufficiently small for $s=n / 2$ and $\nu=2$ in the following (1) and (3).

(1) (NLKG) has a unique local solution $u$ with $\left(u, \partial_{t} u\right) \in C\left([-T, T] ; \mathcal{H}^{s}\right)$ for any given initial data $(\phi, \psi) \in \mathcal{H}^{s}$, where $T$ can be taken depending only on the norm of $(\phi, \psi)$ in $\mathcal{H}^{s^{*}}$.

(2) Let $\nu<2$ if $s=n / 2$. For any given initial data $(\phi, \psi) \in \mathcal{H}^{s}$ let $u \in$ $C\left(\left(-T_{*}, T^{*}\right) ; H^{s}\right)$ be a solution of (NLKG) on the maximal interval of existence $\left(-T_{*}, T^{*}\right), T_{*}, T^{*}>0$. If $T^{*}<\infty\left[\right.$ resp. $\left.T_{*}<\infty\right]$, then $\left\|\left(u(t), \partial_{t} u(t)\right) ; \mathcal{H}^{s^{*}}\right\|$ blows up at $t=T^{*}\left[\right.$ resp. $\left.t=-T_{*}\right]$.

(3) Let $1 \leq p<1+4 / n$. Moreover let $f$ satisfy $N(s,(s-3 / 2+n(p-$ $\left.1) / 4)_{+}, p\right)$ when $1+2 / n \leq p$. Then $T_{*}$ and $T^{*}$ are estimated from below as

$$
T_{*} \wedge T^{*} \geq\left\{\left\|(\phi, \psi) ; \mathcal{H}^{s_{0}}\right\|^{p-1} H\left(\left\|(\phi, \psi) ; \mathcal{H}^{s^{*}}\right\|\right)\right\}^{-4 /(4-n(p-1))},
$$

where $s_{0}$ is a number with $0<s_{0} \leq 1 / 2$, and $H(\cdot)$ is a nonnegative, nondecreasing function on $\mathbb{R}_{+}$. In particular if $p \neq 1$, then $T_{*}$ and $T^{*}$ can be made sufficiently large by taking $\left\|(\phi, \psi) ; \mathcal{H}^{s_{0}}\right\|$ sufficiently small with keeping $\left\|(\phi, \psi) ; \dot{\mathcal{H}}^{s^{*}}\right\|$ bounded.

(4) Let $\gamma_{s^{*}}>0$ be any number, but sufficiently small if $s=n / 2$ and $\nu=2$. Let $p \geq 1+4 / n$. Let $f$ satisfy $N(s, s-1 / 2, p)$. Then there exists $\gamma_{1 / 2}>0$ with the following property.

(4a) For any initial data $(\phi, \psi) \in A^{s}\left(\gamma_{1 / 2}, \gamma_{s^{*}}\right)$, (NLKG) has a unique global solution $u$ in $C\left(\mathbb{R} ; H^{s}\right)$. Moreover the solution $u$ satisfies $\left(u, \partial_{t} u\right) \in$ $C_{b}\left(\mathbb{R} ; \mathcal{H}^{s}\right)$ and there exists unique two pairs $\left(\phi_{+}, \psi_{+}\right)$and $\left(\phi_{-}, \psi_{-}\right)$in $\mathcal{H}^{s}$ such that

$$
\left\|\left(u(t)-v_{ \pm}(t), \partial_{t}\left(u(t)-v_{ \pm}(t)\right)\right) ; \mathcal{H}^{s}\right\| \rightarrow 0 \quad \text { as } \quad t \rightarrow \pm \infty
$$

where $v_{ \pm}(t) \equiv \dot{K}(t) \phi_{ \pm}+K(t) \psi_{ \pm}$.

(4b) For any data $\left(\phi_{-}, \psi_{-}\right) \in A^{s}\left(\gamma_{1 / 2}, \gamma_{s^{*}}\right)$, there exists a unique global solution $u$ of $(\mathrm{NLKG})$ in $C\left(\mathbb{R} ; H^{s}\right)$ and a unique state $\left(\phi_{+}, \psi_{+}\right) \in \mathcal{H}^{s}$ such 
that (1.3) holds. Moreover the scattering operator $S:\left(\phi_{-}, \psi_{-}\right) \mapsto\left(\phi_{+}, \psi_{+}\right)$is well-defined on $A^{s}\left(\gamma_{1 / 2}, \gamma_{s^{*}}\right)$ to $\mathcal{H}^{s}$ and is continuous in the following sense. If $\left\{\left(\phi_{-}^{j}, \psi_{-}^{j}\right)\right\}_{j=1}^{\infty} \subset A^{s}\left(\gamma_{1 / 2}, \gamma_{s^{*}}\right)$ satisfies

$$
\left\|\left(\phi_{-}-\phi_{-}^{j}, \psi_{-}-\psi_{-}^{j}\right) ; \mathcal{H}^{1 / 2}\right\| \rightarrow 0
$$

as $j \rightarrow \infty$, then

$$
\left\|\left(\phi_{+}-\phi_{+}^{j}, \psi_{+}-\psi_{+}^{j}\right) ; \mathcal{H}^{\mu}\right\| \rightarrow 0
$$

as $j \rightarrow \infty$ for any $\mu$ with $\mu<s$, where $\left(\phi_{+}^{j}, \psi_{+}^{j}\right)=S\left(\left(\phi_{-}^{j}, \psi_{-}^{j}\right)\right)$.

(5) The solutions given by (1) and (4a) have the continuous dependence on the initial data in the following sense. For any initial data $(\phi, \psi),\left\{\left(\phi_{j}, \psi_{j}\right)\right\}_{j=1}^{\infty}$ in $A^{s}\left(\gamma_{s_{0}}, \gamma_{s^{*}}\right)$, let $u,\left\{u_{j}\right\}_{j=1}^{\infty}$ be the corresponding solutions of (NLKG) given by (1) or (4a). If $\left\{\left(\phi_{j}, \psi_{j}\right)\right\}_{j=1}^{\infty}$ satisfies

$$
\left\|\left(\phi-\phi_{j}, \psi-\psi_{j}\right) ; \mathcal{H}^{s_{0}}\right\| \rightarrow 0
$$

as $j \rightarrow \infty$, then

$$
\left\|\left(u-u_{j}, \partial_{t}\left(u-u_{j}\right)\right) ; L^{\infty}\left(I ; \mathcal{H}^{\mu}\right)\right\| \rightarrow 0
$$

as $j \rightarrow \infty$ for any $\mu$ with $\mu<s$, where $I=[-T, T]$ for $(1)$ and $I=\mathbb{R}, s_{0}=1 / 2$ for (4a).

Remark 1.1. For $s=n / 2$, the global case (4) in the theorem above covers for instance the nonlinearities of the form

$$
\begin{array}{ll}
f(u)=c\left(e^{\kappa|u|^{2}}-1-\kappa|u|^{2}\right) u & \text { for } n=1, \\
f(u)=c\left(e^{\kappa|u|^{2}}-1\right) u & \text { for } n=2,3, \\
f(u)=c\left(e^{\kappa|u|^{2}}-1\right) & \text { for } n \geq 4,
\end{array}
$$

with $c \in \mathbb{C}, \kappa>0$. To our knowledge, both in the local and global cases there is no other work to treat Klein-Gordon equations with nonlinearity of exponential growth in the $H^{s}$-theory with $s \leq n / 2$. In view of Trudinger's inequality the growth rate as $e^{\kappa|z|^{2}}$ at infinity seems to be optimal at the level of $H^{n / 2}$ (see [25]).

The method of proof of Theorem 1.1 depends on the Strichartz estimates and on the Leibniz type estimate on the composite function $f \circ u$, both of which are described in terms of Besov spaces. The former is given by Corollary 2.1 below, which extends the estimates in [29, Proposition 1.6]. The latter is given by Proposition 3.1. In this paper, different positive constants might be denoted by the same letter $C$. 


\section{§2. Strichartz Estimates for Klein-Gordon Equations}

In order to describe our propositions in concise form, we use the geometric notation, following T. Kato [8]. For any $0 \leq \theta \leq 1$, let $\lambda$ and $\sigma$ be numbers defined by

$$
\lambda \equiv(n+1+\theta) / 2, \quad n-1-\theta \leq \sigma \leq n-1+\theta, \quad \sigma>0 .
$$

Let $\square$ be the closed unit square in $\mathbb{R}^{2}$ defined by $0 \leq x, y \leq 1$. Let $O, A, B, C, D$, $E, F$ be the points, and $T$ and $T_{0}$ be the subsets of $\square$ given by

$$
\begin{aligned}
& O=(0,0), \quad A=(1,1), \quad B=(0,1 / 2), \quad C=(1 / 2,(\sigma-2) / 2 \sigma), \\
& D=(1 / 2,0), \quad E=(1,(\sigma-2) / 2 \sigma), \quad F=(0,(\sigma-2) / 2 \sigma), \\
& (C=(\sigma / 4,0), E=(\sigma / 2,0), F=O \text { if } \sigma<2), \\
& T=\{B\} \cup(B E F), \quad T^{\prime}=\left\{B^{\prime}\right\} \cup\left(B^{\prime} E^{\prime} F^{\prime}\right), \\
& T_{0}=[O B C D] \backslash\{C\}\left(T_{0}=[O B C] \text { if } \sigma<2\right),
\end{aligned}
$$

where $\left(Q_{1} Q_{2}\right),\left(Q_{1} Q_{2} Q_{3}\right),\left(Q_{1} Q_{2} Q_{3} Q_{4}\right)$ denote the interiors of the segment, triangle, quadrangle in $\square$ determined by $\left\{Q_{j}\right\}$ in $\square$, and $\left[Q_{1} Q_{2}\right],\left[Q_{1} Q_{2} Q_{3}\right]$, $\left[Q_{1} Q_{2} Q_{3} Q_{4}\right]$ denotes those closures, respectively. For any $q$ with $1 \leq q \leq \infty$, $q^{\prime}$ denotes the conjugate index of $q$ given by $1 / q+1 / q^{\prime}=1$, and for any $Q=$ $(1 / q, 1 / r)$ in $\square, Q^{\prime}$ denotes the point $Q^{\prime}=\left(1 / q^{\prime}, 1 / r^{\prime}\right)$. For any $Q=(1 / q, 1 / r)$ and $\tilde{Q}=(1 / \tilde{q}, 1 / \tilde{r})$ in $\square$, we call the pair $(Q, \tilde{Q})$ preadmissible pair if

$$
\left\|\int_{J} \frac{U(t-s)}{\omega} h(s) d s ; L^{q}\left(I ; B_{r}^{\rho}\right)\right\| \leq C\left\|h ; L^{\tilde{q}}\left(I ; B_{\tilde{r}}^{\tilde{\rho}}\right)\right\|
$$

for any intervals $I, J \subset \mathbb{R}, h \in L^{\tilde{q}}\left(I ; B_{\tilde{r}}^{\tilde{\rho}}\right)$, and $\rho, \tilde{\rho} \in \mathbb{R}$ with

$$
\rho+n \alpha(r)-2(n-\lambda) / \sigma q=1+2(n-\lambda) / \sigma+\tilde{\rho}+n \alpha(\tilde{r})-2(n-\lambda) / \sigma \tilde{q},
$$

where $\alpha(r) \equiv 1 / 2-1 / r$, the constant $C$ is independent of $I, J, h$, but may be dependent on $\rho$ and $\tilde{\rho}$. For any preadmissible pair $(Q, \tilde{Q})$ we call the pair admissible pair if

$$
\left\|U(t) \phi ; L^{q}\left(I ; B_{r}^{\rho}\right)\right\| \leq C\left\|\phi ; H^{s}\right\|
$$

for any interval $I \subset \mathbb{R}, \phi \in H^{s}$ and $s, \rho \in \mathbb{R}$ with

$$
s=\rho+n \alpha(r)-2(n-\lambda) / \sigma q,
$$

where the constant $C$ is independent of $I, \phi$, but may be dependent on $s$ and $\rho$. 
Proposition 2.1. Let $\tilde{Q}=(1 / \tilde{q}, 1 / \tilde{r})$ in $T^{\prime}$. Let $Q=(1 / q, 1 / r)$ satisfy $1 / q<1 / \tilde{q}$ and

$$
\begin{aligned}
& 1 / \tilde{r}+2 / \sigma \tilde{q}=1 / r+2 / \sigma q+2 / \sigma, \\
& 1 / r+1 / \sigma q<1 / 2,1 / r>(\sigma-2) / \sigma \tilde{r}^{\prime} .
\end{aligned}
$$

Moreover let $1 / q>0$ if $\tilde{Q} \in\left(B^{\prime} C^{\prime} E^{\prime}\right)$. Then $(Q, \tilde{Q})$ is a preadmissible pair.

Remark 2.1. Let $1 \leq \tilde{q}<\infty, 1 \leq \tilde{r}<\infty$ and $\tilde{Q}=(1 / \tilde{q}, 1 / \tilde{r})$. If $(Q, \tilde{Q})$ is a preadmissible pair, then $\left(\tilde{Q}^{\prime}, Q^{\prime}\right)$ is also a preadmissible pair. This follows from duality argument on (2.2).

Remark 2.2. Let $Q \equiv(1 / q, 1 / r), Q_{1} \equiv\left(1 / q, 1 / r_{1}\right)$ in $\square$ with $1 / r_{1} \leq$ $1 / r$. For any preadmissible [resp. admissible] pair $(Q, \tilde{Q}),\left(Q_{1}, \tilde{Q}\right)$ is also a preadmissible [resp. admissible] pair by the embedding $B_{r_{1}}^{\rho_{1}} \hookrightarrow B_{r}^{\rho}$ which is satisfied by $\rho_{1}+n \alpha\left(r_{1}\right)=\rho+n \alpha(r)$.

Proposition 2.2. Let $\sigma>2$. Let $Q \in(C D], \tilde{Q} \in\left(C^{\prime} D^{\prime}\right]$. Then $(Q, \tilde{Q})$ is an admissible pair.

Corollary 2.1. Let $n \geq 1$. Let $Q$ and $\tilde{Q}$ satisfy $Q \in T_{0}$ and $\tilde{Q}^{\prime} \in T_{0}$. Then $(Q, \tilde{Q})$ is an admissible pair.

Proof of Proposition 2.1. Let $\tilde{\psi}$ and $\left\{\psi_{j}\right\}_{j \in \mathbb{Z}}$ be as in the definition of the Besov space in the introduction. We start from following estimates, which are derived from the method of stationary phase (see [3, Theorem 3.2], [6, Appendix]).

$$
\left\|\exp (i t \omega) \tilde{\psi} ; L^{\infty}\right\| \leq C \min \left\{1,|t|^{-n / 2}\right\}
$$

$$
\left\|\exp (i t \omega) \psi_{j} ; L^{\infty}\right\| \leq C 2^{n j} \min \left\{1,\left(2^{j}|t|\right)^{-(n-1) / 2}\right\} \min \left\{1,\left(2^{-j}|t|\right)^{-1 / 2}\right\}
$$

for any $j \geq 1$, where the constant $C$ is independent of $j$ and $t$. Therefore (2.7) is rewritten as

$$
\left\|\exp (i t \omega) \psi_{j} ; L^{\infty}\right\| \leq C 2^{\lambda j}|t|^{-\sigma / 2}
$$

for any $j \geq 1$. We denote by ${ }^{\wedge}$ the Fourier transform $\mathcal{F}$. Since $\hat{\tilde{\psi}}=\left(\hat{\tilde{\psi}}+\hat{\psi}_{1}\right) \hat{\tilde{\psi}}$ and $\hat{\psi}_{j}=\sum_{k=j-1}^{j+1} \hat{\psi}_{k} \hat{\psi}_{j}$, we have

$$
\begin{aligned}
\left\|\tilde{\psi} * \exp (i t \omega) \phi ; L^{\infty}\right\| & \leq\left(\left\|\exp (i t \omega) \tilde{\psi} ; L^{\infty}\right\|+\left\|\exp (i t \omega) \psi_{1} ; L^{\infty}\right\|\right)\left\|\tilde{\psi} * \phi ; L^{1}\right\| \\
& \leq C\left(\min \left\{1,|t|^{-n / 2}\right\}+2^{\lambda}|t|^{-\sigma / 2}\right)\left\|\tilde{\psi} * \phi ; L^{1}\right\| \\
& \leq C|t|^{-\sigma / 2}\left\|\tilde{\psi} * \phi ; L^{1}\right\|,
\end{aligned}
$$




$$
\begin{aligned}
\left\|\psi_{j} * \exp (i t \omega) \phi ; L^{\infty}\right\| & \leq \sum_{k=j-1}^{j+1}\left\|\exp (i t \omega) \psi_{k} ; L^{\infty}\right\|\left\|\psi_{j} * \phi ; L^{1}\right\| \\
& \leq C \sum_{k=j-1}^{j+1} 2^{\lambda k}|t|^{-\sigma / 2}\left\|\psi_{j} * \phi ; L^{1}\right\| \\
& \leq C 2^{\lambda j}|t|^{-\sigma / 2}\left\|\psi_{j} * \phi ; L^{1}\right\|
\end{aligned}
$$

for any $j \geq 1$, where the constant $C$ is independent of $j$ and $\phi$. Therefore by the definition of the Besov space, we have

$$
\left\|U(t) \phi ; B_{\infty}^{0}\right\| \leq C|t|^{-\sigma / 2}\left\|\phi ; B_{1}^{\lambda}\right\| .
$$

Interpolating between (2.8) and the unitarity of $U(t)$ in $L^{2}=B_{2}^{0}$, we also have

$$
\left\|U(t) \phi ; B_{r}^{0}\right\| \leq C|t|^{-\sigma \alpha(r)}\left\|\phi ; B_{r^{\prime}}^{2 \lambda \alpha(r)}\right\|
$$

for any $2 \leq r \leq \infty$. Now let $q_{1}$ and $r_{1}$ satisfy $1<q_{1}<\infty$ and

$$
1 / r_{1}+2 / \sigma q_{1}=1 / 2,1 / r_{1}>(\sigma-2) / 2 \sigma\left(1 / r_{1} \geq 0 \text { if } \sigma<2\right) .
$$

Then applying the Hardy-Littlewood-Sobolev inequality to (2.9) in the time variable, we have

$$
\left\|\int_{J} U(t-s) h(s) d s ; L^{q_{1}}\left(I ; B_{r_{1}}^{-\lambda \alpha\left(r_{1}\right)}\right)\right\| \leq C\left\|h ; L^{q_{1}^{\prime}}\left(I ; B_{r_{1}^{\prime}}^{-\lambda \alpha\left(r_{1}^{\prime}\right)}\right)\right\|
$$

for any intervals $I, J \subset \mathbb{R}$, where we have used the fact that for any $\mu \in \mathbb{R}$ and any $r$ with $1 \leq r \leq \infty$ the operator $\omega^{\mu}$ is an isomorphism on $B_{r}^{0}$ to $B_{r}^{-\mu}$. Especially by the unitarity of $U(t)$ in $L^{2}$ and a duality argument, (2.11) also shows

$$
\left\|\int_{J} U(t-s) h(s) d s ; L^{\infty}\left(I ; B_{2}^{0}\right)\right\| \leq C\left\|h ; L^{q_{1}^{\prime}}\left(I ; B_{r_{1}^{\prime}}^{-\lambda \alpha\left(r_{1}^{\prime}\right)}\right)\right\|
$$

for any intervals $I, J \subset \mathbb{R}$, where we have used $L^{2}=B_{2}^{0}$ with equivalent norms. Interpolating between (2.11) and (2.12), and applying a duality argument on $U$, we obtain for any $\left(1 / q_{j}, 1 / r_{j}\right) \in[B C)\left(\left(1 / q_{j}, 1 / r_{j}\right) \in[B C]\right.$ if $\left.\sigma<2\right), j=1,2$,

$$
\left\|\int_{J} U(t-s) h(s) d s ; L^{q_{1}}\left(I ; B_{r_{1}}^{-\lambda \alpha\left(r_{1}\right)}\right)\right\| \leq C\left\|h ; L^{q_{2}^{\prime}}\left(I ; B_{r_{2}^{\prime}}^{-\lambda \alpha\left(r_{2}^{\prime}\right)}\right)\right\|
$$

for any intervals $I, J \subset \mathbb{R}$. On the other hand, if we consider the dual operator $U^{\prime}$ of $U(\cdot)$ on function spaces of space-time $\mathbb{R}^{1+n}$ of the form

$$
U^{\prime} h \equiv \int_{\mathbb{R}} U(-s) h(s) d s,
$$


then a similar argument on (2.12) shows the inequality

$$
\left\|U^{\prime} h ; B_{2}^{0}\right\| \leq C\left\|h ; L^{q_{1}^{\prime}}\left(I ; B_{r_{1}^{\prime}}^{-\lambda \alpha\left(r_{1}^{\prime}\right)}\right)\right\|
$$

for any interval $I \subset \mathbb{R}$. Applying duality for this inequality, we have

$$
\left\|U(t) \phi ; L^{q_{1}}\left(I ; B_{r_{1}}^{-\lambda \alpha\left(r_{1}\right)}\right)\right\| \leq C\left\|\phi ; B_{2}^{0}\right\|,
$$

where we note that (2.15) also holds for $q_{1}=\infty$ since in the case $q_{1}=\infty, r_{1}$ is equal to 2 , so that the above inequality reduces to the unitary of $U(t)$ in $L^{2}$. Consequently we have obtained the following lemma.

Lemma 2.1. Let $n \geq 1$. Let $\lambda, \sigma$ be as in (2.1). For $j=1,2$, let $\left(1 / q_{j}, 1 / r_{j}\right) \in[B C)\left(\left(1 / q_{j}, 1 / r_{j}\right) \in[B C]\right.$ if $\left.\sigma<2\right)$. Then for any $s \in \mathbb{R}, U$ satisfies the estimates

$$
\begin{gathered}
\left\|U(t) \phi ; L^{q_{1}}\left(I ; B_{r_{1}}^{s-\lambda \alpha\left(r_{1}\right)}\right)\right\| \leq C\left\|\phi ; H^{s}\right\| \\
\left\|\int_{J} \frac{U(t-s)}{\omega} h(s) d s ; L^{q_{1}}\left(I ; B_{r_{1}}^{s-\lambda \alpha\left(r_{1}\right)}\right)\right\| \leq C\left\|h ; L^{q_{2}^{\prime}}\left(I ; B_{r_{2}^{\prime}}^{s-\lambda \alpha\left(r_{2}^{\prime}\right)-1}\right)\right\|
\end{gathered}
$$

for any intervals $I, J \subset \mathbb{R}, \phi \in H^{s}$ and $h \in L^{q_{2}^{\prime}}\left(I ; B_{r_{2}^{\prime}}^{s-\lambda \alpha\left(r_{2}^{\prime}\right)-1}\right)$.

Now let $\tilde{Q}$ and $Q$ be as in the assumption of Proposition 2.1 with $\tilde{Q} \neq B^{\prime}$ and $1 / r \leq 1 / \tilde{r}^{\prime}$. Let $\eta$ be the parameter given by $\eta=-2 \alpha(\tilde{r})$ (so that $0<$ $\eta<1)$, and let $r_{1}=(1-\eta) r, 1 / q_{1}=\sigma \alpha\left(r_{1}\right) / 2$. Then $\left(1 / q_{1}, 1 / r_{1}\right)$ satisfies (2.10). Therefore applying the complex interpolation between (2.8) and (2.15), we have

$$
\left\||t|^{\eta \sigma / 2} U(t) h ; L^{\ell}\left(I ; B_{r}^{-\lambda \alpha(r)}\right)\right\| \leq C\left\|h ; B_{\tilde{r}}^{-\lambda \alpha(\tilde{r})}\right\|,
$$

where $\ell$ is given by $1 / \ell \equiv(1-\eta) / q_{1}$. To estimate the integral operator in space-time as in $(2.2)$, we exploit the standard duality in $(1+n)$-variables of the form

$$
I \equiv\left\langle\int_{J} U(\cdot-s) h(s) d s, h_{1}\right\rangle_{1+n} .
$$

Changing the variable by $\tau=t-s$ and applying duality in space-time to $I$, we have

$$
|I| \leq C \int\left\||\tau|^{\eta \sigma / 2} U(\tau) h(s) ; L_{\tau}^{\ell} B_{r}^{-\lambda \alpha(r)}\right\|\left\|\left.|| \tau\right|^{-\eta \sigma / 2} h_{1}(s+\tau) ; L_{\tau}^{\ell^{\prime}} B_{r^{\prime}}^{\lambda \alpha(r)}\right\| d s,
$$


where $L_{\tau}^{\ell}$ denotes the $L^{\ell}$ norm with respect to the variable $\tau$. By (2.16) and the Hölder inequality in the variable $s$, we have

$$
|I| \leq C\left\|h ; L_{s}^{\tilde{q}} B_{\tilde{r}}^{-\lambda \alpha(\tilde{r})}\right\| \cdot\|\||\tau|^{-\eta \sigma / 2} h_{1}(s+\tau) ; L_{\tau}^{\ell^{\prime}} B_{r^{\prime}}^{\lambda \alpha(r)}\left\|; L_{s}^{\tilde{q}^{\prime}}\right\| .
$$

The second factor on the right hand side of the last inequality is rewritten as

$$
I_{1} \equiv\left\|\int|\tau|^{-\ell^{\prime} \eta \sigma / 2}\right\| h_{1}(s+\tau) ; B_{r^{\prime}}^{\lambda \alpha(r)}\left\|^{\ell^{\prime}} d \tau ; L_{s}^{\tilde{q}^{\prime} / \ell^{\prime}}\right\|^{1 / \ell^{\prime}} .
$$

Now let $r^{*}$ be given by

$$
1 / r^{*}-\ell^{\prime} / \tilde{q}^{\prime}=-\ell^{\prime} \eta \sigma / 2+1
$$

then $1>1 / r^{*}>1 / r^{*}-\ell^{\prime} / \tilde{q}^{\prime}>0$ and $q^{\prime}=\ell^{\prime} r^{*}$. Then we apply the HardyLittlewood-Sobolev inequality to $I_{1}$ to obtain

$$
I_{1} \leq C\|\| h_{1} ; B_{r^{\prime}}^{\lambda \alpha(r)}\left\|^{\ell^{\prime}} ; L_{t}^{r^{*}}\right\|^{1 / \ell^{\prime}}=C\left\|h_{1} ; L_{t}^{q^{\prime}} B_{r^{\prime}}^{\lambda \alpha(r)}\right\| .
$$

So that we have

$$
|I| \leq C\left\|h ; L_{t}^{\tilde{q}} B_{\tilde{r}}^{-\lambda \alpha(\tilde{r})}\right\|\left\|h_{1} ; L_{t}^{q^{\prime}} B_{r^{\prime}}^{\lambda \alpha(r)}\right\|
$$

for any interval $J$. Equivalently we have

$$
\left\|\int_{J} U(t-s) h(s) d s ; L_{t}^{q} B_{r}^{-\lambda \alpha(r)}\right\| \leq C\left\|h ; L_{t}^{\tilde{q}} B_{\tilde{r}}^{-\lambda \alpha(\tilde{r})}\right\|
$$

for any interval $J \subset \mathbb{R}$. Since for any $\mu \in \mathbb{R}, \omega^{\mu}$ is an isomorphism from $B_{r}^{0}$ to $B_{r}^{-\mu}$ with $1 \leq r \leq \infty$, we obtain the required results except for the cases $\tilde{Q}=B^{\prime}$ or $1 / r>1 / \tilde{r}^{\prime}$. In the case $\tilde{Q}=B^{\prime}$, we have obtained the required results by Lemma 2.1. Since the assumptions in Proposition 2.1 hold with $1 / r^{\prime \prime}>1 / \tilde{r}^{\prime}$ for the pair $\left(\tilde{Q}^{\prime}, Q^{\prime}\right)$ in the case $1 / r>1 / \tilde{r}^{\prime}$, by the above argument, we conclude that $\left(\tilde{Q}^{\prime}, Q^{\prime}\right)$ is a preadmissible pair. So that $(Q, \tilde{Q})$ is also a preadmissible pair by Remark 2.1 .

Proof of Proposition 2.2. Let $\tilde{\psi}$ and $\left\{\psi_{j}\right\}_{j \in \mathbb{Z}}$ be as in the definition of the Besov space. We start from (2.6) and (2.7). Let $\lambda, \sigma$ be those in (2.1) with $\sigma>2$. Then

$$
\left\|U(t) \psi_{j} ; L^{\infty}\right\| \leq C \min \left\{2^{n j}, 2^{\lambda j}|t|^{-\sigma / 2}\right\}
$$

for any $j \geq 1$. Since $\hat{\tilde{\psi}}=\left(\hat{\tilde{\psi}}+\hat{\psi}_{1}\right) \hat{\tilde{\psi}}$, we have

$$
\left\|\tilde{\psi} * U(t) \phi ; L^{\infty}\right\| \leq\left(\left\|U(t) \tilde{\psi} ; L^{\infty}\right\|+\left\|U(t) \psi_{1} ; L^{\infty}\right\|\right)\left\|\tilde{\psi} * \phi ; L^{1}\right\| .
$$


Interpolating between the above inequality and the equality $\left\|\tilde{\psi} * U(t) \phi ; L^{2}\right\|=$ $\left\|\tilde{\psi} * \phi ; L^{2}\right\|$, we have

$$
\left\|\tilde{\psi} * U(t) \phi ; L^{r}\right\| \leq\left(\left\|U(t) \tilde{\psi} ; L^{\infty}\right\|^{2 \alpha(r)}+\left\|U(t) \psi_{1} ; L^{\infty}\right\|^{2 \alpha(r)}\right)\left\|\tilde{\psi} * \phi ; L^{r^{\prime}}\right\|
$$

for any $2 \leq r \leq \infty$. If $r$ satisfies $0 \leq 1 / r<(\sigma-2) / 2 \sigma$, then by a direct calculation we have

$$
\|\| U(t) \tilde{\psi} ; L^{\infty}\left\|^{2 \alpha(r)} ; L_{t}^{1}\right\| \leq C n \alpha(r) /(n \alpha(r)-1),
$$

and

$$
\|\| U(t) \psi_{j} ; L^{\infty}\left\|^{2 \alpha(r)} ; L_{t}^{1}\right\| \leq C \sigma \alpha(r) 2^{(2 n \alpha(r) \sigma+2(\lambda-n)) j / \sigma} /(\sigma \alpha(r)-1)
$$

for any $j \geq 1$, where the constant $C$ is independent of $j$. Therefore by the Young inequality, we have

$$
\left\|\tilde{\psi} * \int_{J} U(t-s) h(s) d s ; L_{t}^{2} L^{r}\right\| \leq C\left\|\tilde{\psi} * h ; L_{t}^{2} L^{r^{\prime}}\right\|
$$

for any interval $J \subset \mathbb{R}$, where the constant $C$ is independent of $J$ and $h$. Similarly if we use $\hat{\psi}_{j}=\sum_{k=j-1}^{j+1} \hat{\psi}_{k} \hat{\psi}_{j}$, then we have

$$
\left\|\psi_{j} * \int_{J} U(t-s) h(s) d s ; L_{t}^{2} L^{r}\right\| \leq C 2^{(2 n \alpha(r) \sigma+2(\lambda-n)) j / \sigma}\left\|\psi_{j} * h ; L_{t}^{2} L^{r^{\prime}}\right\|
$$

for any $j \geq 1$ and any interval $J \subset \mathbb{R}$. So that by the definition of the Besov norm, we have

$$
\left\|\int_{J} U(t-s) h(s) d s ; L_{t}^{2} B_{r}^{0}\right\| \leq C\left\|h ; L_{t}^{2} B_{r^{\prime}}^{(2 n \alpha(r) \sigma+2(\lambda-n)) / \sigma}\right\|
$$

for any interval $J \subset \mathbb{R}$. Now let $Q \equiv(1 / q, 1 / r), \tilde{Q} \equiv(1 / \tilde{q}, 1 / \tilde{r})$ with $\tilde{r} \equiv r^{\prime}$, $q=\tilde{q} \equiv 2$. Then since $(2.3)$ is rewritten as

$$
\tilde{\rho}+1-\rho=(2 n \alpha(r) \sigma+2(\lambda-n)) / \sigma,
$$

so that $(2.17)$ shows that $(Q, \tilde{Q})$ is a preadmissible pair. Taking $r$ as $1 / r \rightarrow$ $(\sigma-2) / 2 \sigma$ and considering Remark 2.2 , we conclude $(Q, \tilde{Q})$ is a preadmissible pair for any $Q \in(C D]$ and $\tilde{Q} \in\left(C^{\prime} D^{\prime}\right]$. For these $(Q, \tilde{Q})$, (2.4) with (2.5) follows from the duality for the estimate

$$
\left\|U^{\prime} h ; H^{-s}\right\| \leq\left\|h ; L_{t}^{2} B_{r^{\prime}}^{-\rho}\right\|
$$


which is derived from the unitarity of $U(t)$. So that $(Q, \tilde{Q})$ is also an admissible pair.

Proof of Corollary 2.1. If $\sigma<2$, then the conclusion is obtained by Lemma 2.1. We consider the case $\sigma \geq 2$ in the following. The admissibility of the pair $(Q, \tilde{Q})$ for any $Q \in[B C)$ and $\tilde{Q} \in\left[B^{\prime} C^{\prime}\right)$ is shown by Lemma 2.1, while that of the pair $(Q, \tilde{Q})$ for any $Q \in(C D]$ and $\tilde{Q} \in\left(C^{\prime} D^{\prime}\right]$ is shown by Proposition 2.2. Since $(Q, \tilde{Q})$ is a preadmissible pair for any $Q \in(B C)$ and $\tilde{Q} \in\left[C^{\prime} D^{\prime}\right]$ by Proposition 2.1, while $(B, \tilde{Q})$ is a preadmissible pair for any $\tilde{Q} \in\left(C^{\prime} D^{\prime}\right]$ by the duality and the estimate

$$
\left\|\int U(t-s) h(s) d s ; L_{t}^{2} B_{\tilde{r}^{\prime}}^{0}\right\| \leq C\left\|h ; L_{t}^{2} B_{\tilde{r}}^{\left(2 n \alpha\left(\tilde{r}^{\prime}\right) \sigma+2(\lambda-n)\right) / \sigma}\right\|
$$

for $\tilde{Q} \equiv(1 / \tilde{q}, 1 / \tilde{r})$, where the last inequality is from $(2.17)$, so that $(Q, \tilde{Q})$ is a preadmissible pair for any $Q \in[B C)$ and $\tilde{Q} \in\left(C^{\prime} D^{\prime}\right]$. Since any $Q \in[B C)$ satisfies (2.4) with $(2.5)$ by Lemma $2.1,(Q, \tilde{Q})$ is an admissible pair for any $Q \in[B C)$ and $\tilde{Q} \in\left(C^{\prime} D^{\prime}\right]$. The rest of the proof is completed by a duality of the above results and Remark 2.2 .

\section{§3. Estimates for Nonlinear Terms}

In this section, we show estimates for nonlinear terms in Besov spaces.

Proposition 3.1. Let $s \geq n / 2,0<\mu \leq s, 1 \leq p<\infty$. Let $f$ satisfy $N(s, \mu, p)$. Let $1 \leq \tilde{r}<\infty, 2 \leq r<\infty$. Let $\tilde{r}<r$ if $s=n / 2$. For nonnegative integers $\ell$ and $k$ with $1 \leq k \leq[\mu]+1$ and $p \vee k-1+\nu \ell>0$ let $r_{k}(\ell)$ be a number which satisfies $2 \leq r_{k}(\ell)<\infty$ and

$$
1 / \tilde{r}=(p \vee k-1+\nu \ell) / r_{k}(\ell)+1 / r .
$$

And let $m$ be a number with $2 \leq m \leq \infty$ and $1 / \ell=(p-1) / m+1 / r$. Then for $s=n / 2$ the following estimates hold.

$$
\left\|f(u) ; L^{\tilde{r}}\right\| \leq C \sum_{\substack{\ell=0 \\ p-1+\nu \ell>0}}^{\infty} \frac{\kappa^{\ell}}{\ell !}\left\|u ; L^{r_{1}(\ell)}\right\|^{p-1+\nu \ell}\left\|u ; L^{r}\right\|+C \delta(p-1)\left\|u ; L^{\tilde{r}}\right\|,
$$

$$
\begin{gathered}
\left\|f(u) ; \dot{B}_{\tilde{r}}^{\mu}\right\| \leq C \sum_{\substack{\ell=0 \\
p-1+\nu \ell>0}}^{\infty} \frac{\kappa^{\ell}}{\ell !}\left\|u ; L^{r_{1}(\ell)}\right\|^{p-1+\nu \ell}\left\|u ; \dot{B}_{r}^{\mu}\right\|+C \delta(p-1)\left\|u ; \dot{B}_{\tilde{r}}^{\mu}\right\| \\
\text { if } 0<\mu<1,
\end{gathered}
$$


$(3.4)$

$$
\begin{aligned}
& \left\|f(u) ; \dot{B}_{\tilde{r}}^{\mu}\right\| \\
& \leq\left\{\begin{array}{c}
C \sum_{\ell=0, p-1+\nu \ell>0}^{\infty} \frac{\kappa^{\ell}}{\ell !}\left\|u ; L^{r_{1}(\ell)} \cap \dot{B}_{r_{1}(\ell)}^{0}\right\|^{p-1+\nu \ell}\left\|u ; \dot{B}_{r}^{\mu}\right\| \\
+C \delta(p-1)\left\|u ; \dot{B}_{\tilde{r}}^{\mu}\right\| \quad \text { if } 1 \leq \mu \text { and } \mu<p \\
C \sum_{k=1}^{[\mu]+1} \sum_{\ell=0, p \vee k-1+\nu \ell>0}^{\infty} \frac{\kappa^{\ell}}{\ell !}\left\|u ; L^{r_{k}(\ell)} \cap \dot{B}_{r_{k}(\ell)}^{0}\right\|{ }^{p \vee k-1+\nu \ell}\left\|u ; \dot{B}_{r}^{\mu}\right\| \\
+C \delta(p-1)\left\|u ; \dot{B}_{\tilde{r}}^{\mu}\right\| \quad \text { if } 1 \leq \mu \text { and } \mu \geq p,
\end{array}\right.
\end{aligned}
$$

where $\delta(0)=1$ and $\delta(x)=0$ for $x \neq 0, \kappa$ and $\nu$ are nonnegative constants appearing in the assumption $N(s, \mu, p)$ and $C$ is a constant independent of $u$. And for $s>n / 2$ the following estimates hold.

$$
\left\|f(u) ; L^{\ell}\right\| \leq M\left(\left\|u ; L^{\infty}\right\|\right)\left\|u ; L^{m}\right\|^{p-1}\left\|u ; L^{r}\right\|,
$$

$$
\begin{aligned}
& \left\|f(u) ; \dot{B}_{\ell}^{\mu}\right\| \\
& \leq\left\{\begin{array}{l}
M\left(\left\|u ; L^{\infty}\right\|\right)\left\|u ; L^{m}\right\|^{p-1}\left\|u ; \dot{B}_{r}^{\mu}\right\| \quad \text { if } \quad 0<\mu<2 \\
M\left(\left\|u ; L^{\infty} \cap \dot{B}_{\infty}^{0}\right\|\right)\left\|u ; \dot{B}_{m}^{0}\right\|^{p-1}\left\|u ; \dot{B}_{r}^{\mu}\right\| \quad \text { if } \quad \mu \geq 2, m \neq \infty \\
M\left(\left\|u ; L^{\infty} \cap \dot{B}_{\infty}^{0}\right\|\right)\left\|u ; L^{\infty} \cap \dot{B}_{\infty}^{0}\right\|^{p-1}\left\|u ; \dot{B}_{r}^{\mu}\right\| \quad \text { if } \quad \mu \geq 2, m=\infty
\end{array}\right.
\end{aligned}
$$

where $\left\|u ; L^{\infty} \cap \dot{B}_{\infty}^{0}\right\|=\left\|u ; L^{\infty}\right\| \vee\left\|u ; \dot{B}_{\infty}^{0}\right\|$. Moreover we may replace $\dot{B}$ with $B$ on the RHS of (3.3), (3.4) and (3.6).

Proof of Proposition 3.1. Estimates (3.2), (3.5) follow from the Hölder inequality. And (3.6) is shown in [14, Proposition 1.1]. We show the estimates (3.3) as (A), (3.4) with $1 \leq \mu<2$ as (B), (3.4) with $\mu \geq 2$ as (C) in the following. We use the equivalent norms of the homogeneous Besov space such as

$$
\left\|v ; \dot{B}_{r, m}^{\mu}\right\| \simeq \sum_{|\alpha|=[\mu]}\left\{\int_{0}^{\infty}\left(t^{[\mu]-\mu} \sup _{|y|<t}\left\|\partial^{\alpha} v-\partial^{\alpha} \tau_{y} v ; L^{r}\right\|\right)^{m} d t / t\right\}^{1 / m}
$$

for $0<\mu<\infty$ with $\mu \notin \mathbf{Z}, 1 \leq r, m \leq \infty$, or

(3.8) $\left\|v ; \dot{B}_{r, m}^{\mu}\right\|$

$$
\simeq \sum_{|\alpha|=[\mu]-1}\left\{\int_{0}^{\infty}\left(t^{[\mu]-1-\mu} \sup _{|y|<t}\left\|\partial^{\alpha} v-2 \partial^{\alpha} \tau_{y} v+\partial^{\alpha} \tau_{2 y} v ; L^{r}\right\|\right)^{m} d t / t\right\}^{1 / m}
$$

for $1 \leq \mu<\infty, 1 \leq r, m \leq \infty$, where $\tau_{y}$ denotes the translation by $y \in \mathbb{R}^{n}$ given by $\left(\tau_{y} v\right)(x)=v(y+x)$. We also use the embedding

$$
\dot{B}_{r, m_{1}}^{\mu} \hookrightarrow \dot{B}_{r, m_{2}}^{\mu}
$$


for any $\mu \in \mathbb{R}, 1 \leq r \leq \infty, 1 \leq m_{1}<m_{2} \leq \infty$. We note that the constants appearing to use the equivalent norms for (3.7) and (3.8) and to use embedding for (3.9) are independent of $r, m, m_{1}$ and $m_{2}$ for any fixed $\mu>0$ (see [1, Theorems 6.3.1 and 6.3.2]).

(A) By the Taylor expansion of $M$, we have

$$
\left|f(u)-f\left(\tau_{y} u\right)\right| \leq C \sum_{\ell=0}^{\infty} \frac{\kappa^{\ell}}{\ell !}\left(|u| \vee\left|\tau_{y} u\right|\right)^{p-1+\nu \ell}\left|u-\tau_{y} u\right|,
$$

where the constant $C$ is independent of $u$. Applying the Hölder inequality to the last estimate with the equation

$$
1 / \tilde{r}=(p-1+\nu \ell) / r_{1}(\ell)+1 / r
$$

for $p-1+\nu \ell>0$, we have

$$
\begin{aligned}
& \left\|f(u)-f\left(\tau_{y} u\right) ; L^{\tilde{r}}\right\| \\
\leq & C \sum_{\substack{\ell=0 \\
p-1+\nu \ell>0}}^{\infty} \frac{\kappa^{\ell}}{\ell !}\left\|u ; L^{r_{1}(\ell)}\right\|^{p-1+\nu \ell}\left\|u-\tau_{y} u ; L^{r}\right\|+C \delta(p-1)\left\|u-\tau_{y} u ; L^{\tilde{r}}\right\| .
\end{aligned}
$$

So that by (3.7) we obtain

$$
\left\|f(u) ; \dot{B}_{\tilde{r}}^{\mu}\right\| \leq C \sum_{\substack{\ell=0 \\ p-1+\nu \ell>0}}^{\infty} \frac{\kappa^{\ell}}{\ell !}\left\|u ; L^{r_{1}(\ell)}\right\|^{p-1+\nu \ell}\left\|u ; \dot{B}_{r}^{\mu}\right\|+C \delta(p-1)\left\|u ; \dot{B}_{\tilde{r}}^{\mu}\right\| .
$$

(B) By the Taylor expansion of $M$ with the equation

$$
\begin{aligned}
f(u)-2 f\left(\tau_{y} u\right)+ & f\left(\tau_{2 y} u\right) \\
= & f^{\prime}\left(\tau_{y} u\right)\left(u-2 \tau_{y} u+\tau_{2 y} u\right) \\
& +\int_{0}^{1}\left(f^{\prime}\left(\tau_{y} u+\theta\left(u-\tau_{y} u\right)\right)-f^{\prime}\left(\tau_{y} u\right)\right)\left(u-\tau_{y} u\right) d \theta \\
& +\int_{0}^{1}\left(f^{\prime}\left(\tau_{y} u+\theta\left(\tau_{2 y} u-\tau_{y} u\right)\right)-f^{\prime}\left(\tau_{y} u\right)\right)\left(\tau_{2 y} u-\tau_{y} u\right) d \theta,
\end{aligned}
$$

we have

$$
\begin{aligned}
& (3.10)\left|f(u)-2 f\left(\tau_{y} u\right)+f\left(\tau_{2 y} u\right)\right| \\
& \leq C \sum_{\ell=0}^{\infty} \frac{\kappa^{\ell}}{\ell !}\left|\tau_{y} u\right|^{p-1+\nu \ell}\left|u-2 \tau_{y} u+\tau_{2 y} u\right| \\
& + \begin{cases}C \sum_{\ell=0}^{\infty} \frac{\kappa^{\ell}}{\ell !}\left(|u| \vee\left|\tau_{y} u\right|\right)^{\nu \ell}\left|u-\tau_{y} u\right|^{p} & \text { if } \mu<p<2, \\
C \sum_{\ell=0}^{\infty} \frac{\kappa^{\ell}}{\ell !}\left(|u| \vee\left|\tau_{y} u\right|\right)^{(p-2)_{+}+\nu \ell}\left|u-\tau_{y} u\right|^{2} & \text { otherwise. }\end{cases}
\end{aligned}
$$


Since we have

$$
\begin{aligned}
\left\|f(u) ; \dot{B}_{\tilde{r}}^{\mu}\right\| & \leq C\left\{\int_{0}^{\infty}\left(t^{-\mu} \sup _{|y|<t}\left\|f(u)-2 f\left(\tau_{y} u\right)+f\left(\tau_{2 y} u\right) ; L^{\tilde{r}}\right\|\right)^{2} d t / t\right\}^{1 / 2} \\
& \equiv\|\| f(u)-2 f\left(\tau_{y} u\right)+f\left(\tau_{2 y} u\right) \| \mid
\end{aligned}
$$

by (3.8), denoting by $\Lambda_{1}, \Lambda_{2}, \Lambda_{3}$ the first, second, third term, respectively, on the RHS of (3.10), it suffices to show that ||$\left|\Lambda_{j}\right| \|, 1 \leq j \leq 3$, are estimated by the RHS of (3.4).

For $\Lambda_{1}$ we have

$$
\left\|\mid \Lambda_{1}\right\|\left\|\leq C \sum_{\substack{\ell=0 \\ p-1+\nu \ell>0}}^{\infty} \frac{\kappa^{\ell}}{\ell !}\right\| u ; L^{r_{1}(\ell)}\left\|^{p-1+\nu \ell}\right\| u ; \dot{B}_{r}^{\mu}\|+C \delta(p-1)\| u ; \dot{B}_{\tilde{r}}^{\mu} \|
$$

by the Hölder inequality with $1 / \tilde{r}=(p-1+\nu \ell) / r_{1}(\ell)+1 / r$ for $p-1+\nu \ell>0$.

For $\Lambda_{2}$ let $r(\ell)$ be a number given by

$$
p / r(\ell)=(p-1) / r_{1}(\ell)+1 / r .
$$

Then by the equation $1 / \tilde{r}=\nu \ell / r_{1}(\ell)+p / r(\ell)$, we have

$$
\left\|\mid \Lambda_{2}\right\|\left\|\leq C \sum_{\ell=0}^{\infty} \frac{\kappa^{\ell}}{\ell !}\right\| u ; L^{r_{1}(\ell)}\left\|^{\nu \ell}\right\| u ; \dot{B}_{r(\ell), 2 p}^{\mu / p} \|^{p},
$$

where we have used

$$
\left\{\int_{0}^{\infty}\left(t^{-\mu} \sup _{|y|<t}\left\|u-\tau_{y} u ; L^{r(\ell)}\right\|^{p}\right)^{2} d t / t\right\}^{1 / 2} \leq C\left\|u ; \dot{B}_{r(\ell), 2 p}^{\mu / p}\right\|^{p} .
$$

By the convex inequality

$$
\left\|u ; \dot{B}_{r(\ell), 2 p}^{\mu / p}\right\| \leq\left\|u ; \dot{B}_{r_{1}(\ell), \infty}^{0}\right\|^{1-1 / p}\left\|u ; \dot{B}_{r, 2}^{\mu}\right\|^{1 / p}
$$

and the embedding (3.9), we obtain

$$
\left\|\left|\Lambda_{2}\left\|\mid \leq C \sum_{\ell=0}^{\infty} \frac{\kappa^{\ell}}{\ell !}\right\| u ; L^{r_{1}(\ell)} \cap \dot{B}_{r_{1}(\ell)}^{0}\left\|^{p-1+\nu \ell}\right\| u ; \dot{B}_{r}^{\mu} \| .\right.\right.
$$

For $\Lambda_{3}$ let $r(\ell)$ be a number given by the equation

$$
2 / r(\ell)=1 / r_{2}(\ell)+1 / r \text {. }
$$


Then by the equation $1 / \tilde{r}=\left((p-2)_{+}+\nu \ell\right) / r_{2}(\ell)+2 / r(\ell)$, we have

$$
\left\|\left|\Lambda_{3}\left\|\mid \leq C \sum_{\ell=0}^{\infty} \frac{\kappa^{\ell}}{\ell !}\right\| u ; L^{r_{2}(\ell)}\left\|^{(p-2)_{+}+\nu \ell}\right\| u ; \dot{B}_{r(\ell), 4}^{\mu / 2} \|^{2},\right.\right.
$$

where we have used

$$
\left\{\int_{0}^{\infty}\left(t^{-\mu} \sup _{|y|<t}\left\|u-\tau_{y} u ; L^{r(\ell)}\right\|^{2}\right)^{2} d t / t\right\}^{1 / 2} \leq C\left\|u ; \dot{B}_{r(\ell), 4}^{\mu / 2}\right\| .
$$

By the convex inequality

$$
\left\|u ; \dot{B}_{r(\ell), 4}^{\mu / 2}\right\| \leq\left\|u ; \dot{B}_{r_{2}(\ell), \infty}^{0}\right\|^{1 / 2}\left\|u ; \dot{B}_{r, 2}^{\mu}\right\|^{1 / 2}
$$

and (3.9), we obtain

$$
\left\|\Lambda_{3}\right\| \mid \leq C \sum_{\ell=0}^{\infty} \frac{\kappa^{\ell}}{\ell !}\left\|u ; L^{r_{2}(\ell)} \cap \dot{B}_{r_{2}(\ell)}^{0}\right\|^{p \vee 2-1+\nu \ell}\left\|u ; \dot{B}_{r}^{\mu}\right\| .
$$

We note that $\Lambda_{3}$ is considered when $p \notin(\mu, 2)$.

(C) We have

$$
\begin{gathered}
\left\|f(u) ; \dot{B}_{\tilde{r}}^{\mu}\right\| \simeq \sum_{|\alpha|=[\mu]-1} \\
\left\{\int _ { 0 } ^ { \infty } \left(t^{[\mu]-1-\mu} \sup _{|y|<t} \| \partial^{\alpha}(f(u))-2 \partial^{\alpha}\left(f\left(\tau_{y} u\right)\right)\right.\right. \\
\left.\left.+\partial^{\alpha}\left(f\left(\tau_{2 y} u\right)\right) ; L^{\tilde{r}} \|\right)^{2} d t / t\right\}^{1 / 2}
\end{gathered}
$$

for any given $\mu \geq 1$ and $1 \leq r \leq \infty$. We have

$$
\partial^{\alpha}(f(u))=\sum_{k=1}^{[\mu]-1} \sum_{\substack{\beta_{1}+\ldots+\beta_{k}=\alpha \\\left|\beta_{j}\right| \geq 1,1 \leq j \leq k}} C\left([\mu]-1, k,\left\{\beta_{j}\right\}\right) f^{(k)}(u) \prod_{j=1}^{k} \partial^{\beta_{j}} u
$$

where the constant $C\left([\mu]-1, k,\left\{\beta_{j}\right\}\right)$ depends on $[\mu]-1, k$ and $\beta_{j}, 1 \leq j \leq k$, but not on $u$ and $f$. We put $\left\{u_{j}^{k}, v_{j}^{k}, w_{j}^{k}\right\}_{j=0}^{k}$ for $1 \leq k \leq[\mu]-1$ as

$$
\begin{aligned}
& u_{0}^{k}=f^{(k)}(u), \quad v_{0}^{k}=f^{(k)}\left(\tau_{y} u\right), \quad w_{0}^{k}=f^{(k)}\left(\tau_{2 y} u\right), \\
& u_{j}^{k}=\partial^{\beta_{j}} u, \quad v_{j}^{k}=\partial^{\beta_{j}} \tau_{y} u, \quad w_{j}^{k}=\partial^{\beta_{j}} \tau_{2 y} u,
\end{aligned}
$$

then we have

$$
\begin{aligned}
\partial^{\alpha}(f(u)) & -2 \partial^{\alpha}\left(f\left(\tau_{y} u\right)\right)+\partial^{\alpha}\left(f\left(\tau_{2 y} u\right)\right) \\
& =\sum_{k=1}^{\alpha} \sum_{\substack{\beta_{1}+\ldots+\beta_{k}=\alpha \\
\left|\beta_{j}\right| \geq 1,1 \leq j \leq k}} C\left([\mu]-1, k,\left\{\beta_{j}\right\}\right)\left(\prod_{j=0}^{k} u_{j}^{k}-2 \prod_{j=0}^{k} v_{j}^{k}+\prod_{j=0}^{k} w_{j}^{k}\right) .
\end{aligned}
$$


In the following we proceed our argument with $1 \leq k \leq[\mu]-1$ fixed, and remove $k$ of $u_{j}^{k}, v_{j}^{k}, w_{j}^{k}$ for simplicity. We have

$$
\begin{aligned}
& \prod_{j=0}^{k} u_{j}-2 \prod_{j=0}^{k} v_{j}+\prod_{j=0}^{k} w_{j} \\
& =\left(u_{0}-2 v_{0}+w_{0}\right) \prod_{j=1}^{k} u_{j}+\sum_{\imath=1}^{k}\left(v_{0}-w_{0}\right)\left(u_{\imath}-v_{\imath}\right) \prod_{j=1, j<\imath}^{k} u_{j} \prod_{j=1, j>\imath}^{k} v_{j} \\
& +\sum_{\imath=1}^{k}\left(v_{0}-w_{0}\right)\left(v_{\imath}-w_{\imath}\right) \prod_{j=1, j<\imath}^{k} v_{j} \prod_{j=1, j>\imath}^{k} w_{j} \\
& +v_{0}\left(\prod_{j=1}^{k} u_{j}-2 \prod_{j=1}^{k} v_{j}+\prod_{j=1}^{k} w_{j}\right) .
\end{aligned}
$$

Moreover the last term on the RHS of the above equation is rewritten as

$$
\begin{aligned}
v_{0}\left(u_{1}-2 v_{1}+w_{1}\right) \prod_{j=2}^{k} u_{j} & +\sum_{i=2}^{k} v_{0}\left(v_{1}-w_{1}\right)\left(u_{i}-v_{i}\right) \prod_{j=2, j<i}^{k} u_{j} \prod_{j=2, j>i}^{k} v_{j} \\
& +\sum_{i=2}^{k} v_{0}\left(v_{1}-w_{1}\right)\left(v_{i}-w_{i}\right) \prod_{j=2, j<i}^{k} v_{j} \prod_{j=2, j>i}^{k} w_{j} \\
& +v_{0} v_{1}\left(\prod_{j=2}^{k} u_{j}-2 \prod_{j=2}^{k} v_{j}+\prod_{j=2}^{k} w_{j}\right) .
\end{aligned}
$$

With repeated use of the same calculation, the LHS of (3.15) is rewritten as a linear combination of the terms such as

$$
\begin{aligned}
& A_{1} \equiv\left(u_{0}-2 v_{0}+w_{0}\right) \prod_{j=1}^{k} u_{j}, \quad A_{2} \equiv\left(u_{0}-v_{0}\right)\left(u_{1}-v_{1}\right) \prod_{j=2}^{k} v_{j} \\
& A_{3} \equiv v_{0}\left(u_{1}-2 v_{1}+w_{1}\right) \prod_{j=2}^{k} u_{j}, \quad A_{4} \equiv v_{0}\left(v_{1}-w_{1}\right)\left(u_{2}-v_{2}\right) \prod_{j=3}^{k} v_{j}
\end{aligned}
$$

where $\prod_{j=m_{1}}^{m_{2}} z_{j}$ is disregarded for $m_{2}<m_{1}$, and $A_{4}$ appears only for $k \geq 2$. It is sufficient to prove that

$$
\left\|\left|A_{j}\right|\right\| \equiv\left\{\int_{0}^{\infty}\left(t^{[\mu]-1-\mu} \sup _{|y|<t}\left\|A_{j} ; L^{\tilde{r}}\right\|\right)^{2} d t / t\right\}^{1 / 2}
$$

is dominated by the RHS of (3.4) for any $j=1,2,3,4$, since other terms similar to $A_{j}, j=1,2,3,4$, are estimated quite analogously. 
For $A_{1}$, we have

$$
\begin{aligned}
u_{0}- & 2 v_{0}+w_{0} \\
= & f^{(k)}(u)-2 f^{(k)}\left(\tau_{y} u\right)+f^{(k)}\left(\tau_{2 y} u\right) \\
= & f^{(k+1)}\left(\tau_{y} u\right)\left(u-2 \tau_{y} u+\tau_{2 y} u\right) \\
& +\int_{0}^{1}\left(f^{(k+1)}\left(\tau_{y} u+\eta\left(u-\tau_{y} u\right)\right)-f^{(k+1)}\left(\tau_{y} u\right)\right) d \eta\left(u-\tau_{y} u\right) \\
& +\int_{0}^{1}\left(f^{(k+1)}\left(\tau_{y} u+\eta\left(\tau_{2 y} u-\tau_{y} u\right)\right)-f^{(k+1)}\left(\tau_{y} u\right)\right) d \eta\left(\tau_{2 y} u-\tau_{y} u\right) .
\end{aligned}
$$

Therefore by the definition of $f$, we have

$$
\begin{aligned}
\left|u_{0}-2 v_{0}+w_{0}\right| \leq & C\left|\tau_{y} u\right|^{(p-k-1)_{+}}\left|u-2 \tau_{y} u+\tau_{2 y} u\right| M\left(\left|\tau_{y} u\right|\right) \\
& + \begin{cases}C \Lambda_{1} & \text { if } k+1=[\mu] \text { and } \mu<p<[\mu]+1, \\
C \Lambda_{2} & \text { otherwise, }\end{cases}
\end{aligned}
$$

where

$$
\begin{aligned}
\Lambda_{1} \equiv & \left|u-\tau_{y} u\right|^{p-[\mu]+1} M\left(|u| \vee\left|\tau_{y} u\right|\right) \\
& +\left|\tau_{y} u-\tau_{2 y} u\right|^{p-[\mu]+1} M\left(\left|\tau_{y} u\right| \vee\left|\tau_{2 y} u\right|\right) \\
\Lambda_{2} \equiv & \left(|u| \vee\left|\tau_{y} u\right|\right)^{(p-k-2)_{+}}\left|u-\tau_{y} u\right|^{2} M\left(|u| \vee\left|\tau_{y} u\right|\right) \\
& +\left(\left|\tau_{y} u\right| \vee\left|\tau_{2 y} u\right|\right)^{(p-k-2)_{+}}\left|\tau_{y} u-\tau_{2 y} u\right|^{2} M\left(\left|\tau_{y} u\right| \vee\left|\tau_{2 y} u\right|\right)
\end{aligned}
$$

We estimate $A_{1}$ by considering contributions of $A_{1}^{1}, A_{1}^{2}, A_{1}^{3}$ separately, where

$$
\begin{aligned}
& A_{1}^{1} \equiv\left|\tau_{y} u\right|^{(p-k-1)_{+}}\left|u-2 \tau_{y} u+\tau_{2 y} u\right| M\left(\left|\tau_{y} u\right|\right) \prod_{j=1}^{k}\left|\partial^{\beta_{j}} u\right| \\
& A_{1}^{2} \equiv\left|u-\tau_{y} u\right|^{p-[\mu]+1} M\left(|u| \vee\left|\tau_{y} u\right|\right) \prod_{j=1}^{k}\left|\partial^{\beta_{j}} u\right| \\
& A_{1}^{3} \equiv\left(|u|+\left|\tau_{y} u\right|\right)^{(p-k-2)_{+}}\left|u-\tau_{y} u\right|^{2} M\left(|u| \vee\left|\tau_{y} u\right|\right) \prod_{j=1}^{k}\left|\partial^{\beta_{j}} u\right|
\end{aligned}
$$

and we show that $\left\|\mid A_{1}^{j}\right\| \|, j=1,2,3$, are dominated by the RHS of (3.4), so that the required estimate on $\|\left|A_{1}\right|||$ follows. 
We also have

$$
\begin{aligned}
& \left|A_{2}\right| \leq C\left(|u| \vee\left|\tau_{y} u\right|\right)^{(p-k-1)_{+}}\left|u-\tau_{y} u\right|\left|\partial^{\beta_{1}} u-\partial^{\beta_{1}} \tau_{y} u\right| \prod_{j=2}^{k}\left|\partial^{\beta_{j}} \tau_{y} u\right| \\
& \cdot M\left(|u| \vee\left|\tau_{y} u\right|\right), \\
& \left|A_{3}\right| \leq C\left|\tau_{y} u\right|^{(p-k)_{+}}\left|\partial^{\beta_{1}} u-2 \partial^{\beta_{1}} \tau_{y} u+\partial^{\beta_{1}} \tau_{2 y} u\right| \prod_{j=2}^{k}\left|\partial^{\beta_{j}} u\right| M\left(\left|\tau_{y} u\right|\right) \\
& \left|A_{4}\right| \leq C\left|\tau_{y} u\right|^{(p-k)_{+}}\left|\partial^{\beta_{1}} \tau_{y} u-\partial^{\beta_{1}} \tau_{2 y} u\right|\left|\partial^{\beta_{2}} u-\partial^{\beta_{2}} \tau_{y} u\right| \prod_{j=3}^{k}\left|\partial^{\beta_{j}} u\right| M\left(\left|\tau_{y} u\right|\right),
\end{aligned}
$$

where the constant $C$ is independent of $u$. In the following, $\theta_{j}, r_{j}, j=$ $0,1, \ldots, k$, are defined respectively from the case $A_{1}^{1}$ to $A_{4}$.

For $A_{1}^{1}$, by the Taylor expansion of $M$, we have

$$
\left|A_{1}^{1}\right| \leq C \sum_{\ell=0}^{\infty} \frac{\kappa^{\ell}}{\ell !}\left|\tau_{y} u\right|^{(p-k-1)_{+}+\nu \ell}\left|u-2 \tau_{y} u+\tau_{2 y} u\right| \prod_{j=1}^{k}\left|\partial^{\beta_{j}} u\right|,
$$

where the constant $C$ is independent of $u$. Let $\theta_{j}, r_{j}$ be given by

$$
\begin{aligned}
\theta_{0} & \equiv(\mu-[\mu]+1) / \mu, \\
\theta_{j} & \equiv\left|\beta_{j}\right| / \mu \text { for } 1 \leq j \leq k, \\
1 / r_{j} & \equiv\left(1-\theta_{j}\right) / r_{k+1}(\ell)+\theta_{j} / r \quad \text { for } \quad 0 \leq j \leq k .
\end{aligned}
$$

Then

$$
1 / \tilde{r}=\left((p-k-1)_{+}+\nu \ell\right) / r_{k+1}(\ell)+\sum_{j=0}^{k} 1 / r_{j} .
$$

Therefore applying the Hölder inequality, we have

$$
\begin{aligned}
\left\|A_{1}^{1}\right\| \mid \leq & C \sum_{\ell=0}^{\infty} \frac{\kappa^{\ell}}{\ell !}\left\|u ; L^{r_{k+1}(\ell)}\right\|^{(p-k-1)_{+}+\nu \ell} \prod_{j=1}^{k}\left\|\partial^{\beta_{j}} u ; L^{r_{j}}\right\| \\
& \cdot\left\{\int_{0}^{\infty}\left(t^{[\mu]-1-\mu} \sup _{|y|<t}\left\|u-2 \tau_{y} u+\tau_{2 y} u ; L^{r_{0}}\right\|\right)^{2} d t / t\right\}^{1 / 2} .
\end{aligned}
$$

By (3.8), we have

$$
\left\{\int_{0}^{\infty}\left(t^{[\mu]-1-\mu} \sup _{|y|<t}\left\|u-2 \tau_{y} u+\tau_{2 y} u ; L^{r_{0}}\right\|\right)^{2} d t / t\right\}^{1 / 2} \leq C\left\|u ; \dot{B}_{r_{0}}^{\mu \theta_{0}}\right\|,
$$


and by the embedding $\dot{B}_{r_{j}}^{\left|\beta_{j}\right|} \hookrightarrow \dot{H}_{r_{j}}^{\left|\beta_{j}\right|}$, we also have

$$
\left\|\partial^{\beta_{j}} u ; L^{r_{j}}\right\| \leq C\left\|u ; \dot{B}_{r_{j}}^{\mu \theta_{j}}\right\|
$$

for $1 \leq j \leq k$, where the constant $C$ in these inequalities is independent of $\ell$ since $\theta_{j}$ with $0 \leq j \leq k$ are in a compact set in $(0,1)$ and therefore $r_{j}$ with $0 \leq j \leq k$ are also in a compact set in $(1, \infty)$ because of $r \neq \infty$. Applying the convex inequalities such as

$$
\left\|u ; \dot{B}_{r_{j}}^{\mu \theta_{j}}\right\| \leq\left\|u ; \dot{B}_{r_{k+1}(\ell)}^{0}\right\|^{1-\theta_{j}}\left\|u ; \dot{B}_{r}^{\mu}\right\|^{\theta_{j}}
$$

for $0 \leq j \leq k$, we have

$$
\left\|A _ { 1 } ^ { 1 } \left|\left\|\mid \leq C \sum_{\ell=0}^{\infty} \frac{\kappa^{\ell}}{\ell !}\right\| u ; L^{r_{k+1}(\ell)} \cap \dot{B}_{r_{k+1}(\ell)}^{0}\left\|^{p \vee(k+1)-1+\nu \ell}\right\| u ; \dot{B}_{r}^{\mu} \|,\right.\right.
$$

where we have used $\sum_{j=0}^{k} \theta_{j}=1$. Since $k+1 \leq[\mu]$, this is one of the estimates that we have required.

For $A_{1}^{2}$, we first note that $A_{1}^{2}$ is considered only for the case $k+1=[\mu]$ and $\mu<p<[\mu]+1$. Therefore $p \neq 1$, so that $p-1+\nu \ell>0$ and we have $r_{1}(\ell)=\cdots=r_{[\mu]-1}(\ell)$ in (3.1). Let $\theta_{j}, r_{j}$ be given by

$$
\begin{aligned}
\theta_{0} & \equiv(\mu-[\mu]+1) / \mu(p-[\mu]+1), \\
\theta_{j} & \equiv\left|\beta_{j}\right| / \mu \quad \text { for } \quad 1 \leq j \leq k, \\
1 / r_{j} & \equiv\left(1-\theta_{j}\right) / r_{1}(\ell)+\theta_{j} / r \text { for } 0 \leq j \leq k .
\end{aligned}
$$

Then

$$
1 / \tilde{r}=\nu \ell / r_{1}(\ell)+\sum_{j=1}^{k} 1 / r_{j}+(p-[\mu]+1) / r_{0}
$$

so that we have

$$
\begin{aligned}
\left\|\left|A_{1}^{2}\right|\right\| \leq & C \sum_{\ell=0}^{\infty} \frac{\kappa^{\ell}}{\ell !}\left\|u ; L^{r_{1}(\ell)}\right\|^{\nu \ell} \prod_{j=1}^{k}\left\|\partial^{\beta_{j}} u ; L^{r_{j}}\right\| \\
& \cdot\left\{\int_{0}^{\infty}\left(t^{[\mu]-1-\mu} \sup _{|y|<t}\left\|u-\tau_{y} u ; L^{r_{0}}\right\|^{p-[\mu]+1}\right)^{2} d t / t\right\}^{1 / 2} .
\end{aligned}
$$

By (3.7) with

$$
0<(\mu-[\mu]+1) /(p-[\mu]+1)=\theta_{0} \mu<1,
$$


we have

$$
\begin{aligned}
\left\{\int _ { 0 } ^ { \infty } \left(t^{[\mu]-1-\mu} \sup _{|y|<t} \| u-\tau_{y} u ; L^{r_{0}}\right.\right. & \left.\left.\|{ }^{p-[\mu]+1}\right)^{2} d t / t\right\}^{1 / 2} \\
& \leq C\left\|u ; \dot{B}_{r_{0}, 2(p-[\mu]+1)}^{\theta_{0} \mu}\right\|^{p-[\mu]+1} .
\end{aligned}
$$

By (3.9) with $2(p-[\mu]+1) \geq 2$, we have

$$
\left\|u ; \dot{B}_{r_{0}, 2(p-[\mu]+1)}^{\mu \theta_{0}}\right\| \leq C\left\|u ; \dot{B}_{r_{0}}^{\mu \theta_{0}}\right\|
$$

where the constant $C$ in the last two inequalities are independent of $\ell$ since $\theta_{0}$ is in a compact set in $(0,1)$. We also have the estimates of the form (3.16) for $1 \leq j \leq k$. Applying the convex inequalities such as

$$
\left\|u ; \dot{B}_{r_{j}}^{\mu \theta_{j}}\right\| \leq\left\|u ; \dot{B}_{r_{1}(\ell)}^{0}\right\|^{1-\theta_{j}}\left\|u ; \dot{B}_{r}^{\mu}\right\|^{\theta_{j}}
$$

for $0 \leq j \leq k$, we have

$$
\left\|A_{1}^{2}\right\| \mid \leq C \sum_{\ell=0}^{\infty} \frac{\kappa^{\ell}}{\ell !}\left\|u ; L^{r_{1}(\ell)} \cap \dot{B}_{r_{1}(\ell)}^{0}\right\|^{p-1+\nu \ell}\left\|u ; \dot{B}_{r}^{\mu}\right\|,
$$

where we have used $\theta_{0}(p-[\mu]+1)+\sum_{j=1}^{k} \theta_{j}=1$. Since $A_{1}^{2}$ is considered only for the case $\mu<p$, the above inequality is one of the estimates that we have required.

For $A_{1}^{3}$, let $\theta_{j}, r_{j}$ be given by

$$
\begin{aligned}
\theta_{0} & \equiv(\mu-[\mu]+1) / 2 \mu, \\
\theta_{j} & \equiv\left|\beta_{j}\right| / \mu \quad \text { for } \quad 1 \leq j \leq k, \\
1 / r_{j} & \equiv\left(1-\theta_{j}\right) / r_{k+2}(\ell)+\theta_{j} / r \quad \text { for } \quad 0 \leq j \leq k .
\end{aligned}
$$

Then

$$
1 / \tilde{r}=\left((p-k-2)_{+}+\nu \ell\right) / r_{k+2}(\ell)+\sum_{j=1}^{k} 1 / r_{j}+2 / r_{0},
$$

so that we have

$$
\begin{aligned}
\left\|\left|A_{1}^{3} \|\right| \leq C\right. & \sum_{\ell=0}^{\infty} \frac{\kappa^{\ell}}{\ell !}\left\|u ; L^{r_{k+2}(\ell)}\right\|^{(p-k-2)_{+}+\nu \ell} \prod_{j=1}^{k}\left\|\partial^{\beta_{j}} u ; L^{r_{j}}\right\| \\
& \cdot\left\{\int_{0}^{\infty}\left(t^{[\mu]-1-\mu} \sup _{|y|<t}\left\|u-\tau_{y} u ; L^{r_{0}}\right\|^{2}\right)^{2} d t / t\right\}^{1 / 2} .
\end{aligned}
$$


By (3.7) with

$$
0<(\mu-[\mu]+1) / 2=\theta_{0} \mu<1
$$

we have

$$
\left\{\int_{0}^{\infty}\left(t^{[\mu]-1-\mu} \sup _{|y|<t}\left\|u-\tau_{y} u ; L^{r_{0}}\right\|^{2}\right)^{2} d t / t\right\}^{1 / 2} \leq C\left\|u ; \dot{B}_{r_{0}, 4}^{\theta_{0} \mu}\right\|^{2} \leq C\left\|u ; \dot{B}_{r_{0}}^{\theta_{0} \mu}\right\|^{2},
$$

where we have used (3.9) for the last inequality, and the constant $C$ is independent of $\ell$ since $\theta_{0}$ is in a compact set in $(0,1)$. We also have the estimates of the form (3.16) for $1 \leq j \leq k$. Applying the convex inequalities such as

$$
\left\|u ; \dot{B}_{r_{j}}^{\mu \theta_{j}}\right\| \leq\left\|u ; \dot{B}_{r_{k+2}(\ell)}^{0}\right\|^{1-\theta_{j}}\left\|u ; \dot{B}_{r}^{\mu}\right\|^{\theta_{j}}
$$

for $0 \leq j \leq k$, we have

$$
\left\|\left|A_{1}^{3}\right|\right\| \mid \leq C \sum_{\ell=0}^{\infty} \frac{\kappa^{\ell}}{\ell !}\left\|u ; L^{r_{k+2}(\ell)} \cap \dot{B}_{r_{k+2}(\ell)}^{0}\right\|^{p \vee(k+2)-1+\nu \ell}\left\|u ; \dot{B}_{r}^{\mu}\right\| .
$$

Since $k+2 \leq[\mu]+1$, this is one of the estimates that we have required. We note that $A_{1}^{3}$ is not referred for the case $k=[\mu]-1$ and $\mu<p<[\mu]+1$.

For $A_{2}$, let $\theta_{j}, r_{j}$ be given by

$$
\begin{aligned}
\theta_{0} & \equiv(\mu-[\mu]+1) / 2 \mu, \quad \theta_{1} \equiv\left(\mu-[\mu]+1+2 \beta_{1}\right) / 2 \mu, \\
\theta_{j} & \equiv\left|\beta_{j}\right| / \mu, \quad \text { for } \quad 2 \leq j \leq k \\
1 / r_{j} & \equiv\left(1-\theta_{j}\right) / r_{k+1}(\ell)+\theta_{j} / r \quad \text { for } \quad 0 \leq j \leq k .
\end{aligned}
$$

Then

$$
1 / \tilde{r}=\left((p-k-1)_{+}+\nu \ell\right) / r_{k+1}(\ell)+\sum_{j=0}^{k} 1 / r_{j}
$$

so that we have

$$
\begin{aligned}
\left\|A_{2}\right\| \mid \leq & C \sum_{\ell=0}^{\infty} \frac{\kappa^{\ell}}{\ell !}\left\|u ; L^{r_{k+1}(\ell)}\right\|{ }^{(p-k-1)_{+}+\nu \ell} \prod_{j=2}^{k}\left\|\partial^{\beta_{j}} u ; L^{r_{j}}\right\| \\
& \cdot\left\{\int_{0}^{\infty}\left(t^{[\mu]-1-\mu} \sup _{|y|<t}\left\|u-\tau_{y} u ; L^{r_{0}}\right\|\left\|\partial^{\beta_{1}} u-\partial^{\beta_{1}} \tau_{y} u ; L^{r_{1}}\right\|\right)^{2} d t / t\right\}^{1 / 2} .
\end{aligned}
$$


By the Hölder inequality and (3.7), we have

$$
\begin{gathered}
\left\{\int_{0}^{\infty}\left(t^{[\mu]-1-\mu} \sup _{|y|<t}\left\|u-\tau_{y} u ; L^{r_{0}}\right\|\left\|\partial^{\beta_{1}} u-\partial^{\beta_{1}} \tau_{y} u ; L^{r_{1}}\right\|\right)^{2} d t / t\right\}^{1 / 2} \\
\leq C \prod_{j=0}^{1}\left\|u ; \dot{B}_{r_{j}, 4}^{\mu \theta_{j}}\right\| \leq C \prod_{j=0}^{1}\left\|u ; \dot{B}_{r_{j}}^{\mu \theta_{j}}\right\|
\end{gathered}
$$

where we have used (3.9) for the last inequality, and the constant $C$ is independent of $\ell$ since $\theta_{j}, j=0,1$, are in a compact set in $(0,1)$. We also have the estimates of the form (3.16) for $2 \leq j \leq k$. Applying the convex inequalities such as

$$
\left\|u ; \dot{B}_{r_{j}}^{\mu \theta_{j}}\right\| \leq\left\|u ; \dot{B}_{r_{k+1}(\ell)}^{0}\right\|^{1-\theta_{j}}\left\|u ; \dot{B}_{r}^{\mu}\right\|^{\theta_{j}}
$$

for $1 \leq j \leq k$, we have

$$
\left\|A_{2}\right\|\left\|\leq C \sum_{\ell=0}^{\infty} \frac{\kappa^{\ell}}{\ell !}\right\| u ; L^{r_{k+1}(\ell)} \cap \dot{B}_{r_{k+1}(\ell)}^{0}\left\|^{p \vee(k+1)-1+\nu \ell}\right\| u ; \dot{B}_{r}^{\mu} \| .
$$

Since $k+1 \leq[\mu]$, this is one of the estimates that we have required.

For $A_{3}$, let $\theta_{j}, j=1, \ldots, k$, be given by

$$
\begin{aligned}
& \theta_{1} \equiv\left(\mu-[\mu]+1+\beta_{1}\right) / \mu, \\
& \theta_{j} \equiv\left|\beta_{j}\right| / \mu, \quad \text { for } \quad 2 \leq j \leq k .
\end{aligned}
$$

And for $1 \leq j \leq k$ let

$$
1 / r_{j} \equiv \begin{cases}1 / \tilde{r} & \text { if } p=k=1 \text { and } \ell=0 \\ \left(1-\theta_{j}\right) / r_{k}(\ell)+\theta_{j} / r & \text { otherwise. }\end{cases}
$$

Then

$$
1 / \tilde{r}= \begin{cases}1 / r_{1} & \text { if } p=k=1 \text { and } \ell=0 \\ \left((p-k)_{+}+\nu \ell\right) / r_{k}(\ell)+\sum_{j=1}^{k} 1 / r_{j} & \text { otherwise }\end{cases}
$$

so that we have

$$
\begin{gathered}
\left\|A_{3}\right\| \mid \leq C\left(\sum_{\substack{\ell=0 \\
p \vee k-1+\nu \ell>0}}^{\infty} \frac{\kappa^{\ell}}{\ell !}\left\|u ; L^{r_{k}(\ell)}\right\|^{(p-k)_{+}+\nu \ell} \prod_{j=2}^{k}\left\|\partial^{\beta_{j}} u ; L^{r_{j}}\right\|\right. \\
+\delta((p-1) \vee(k-1))) \cdot \Lambda_{3},
\end{gathered}
$$


where $\delta(0)=1, \delta(x)=0$ for $x \neq 0$, and

$$
\Lambda_{3} \equiv\left\{\int_{0}^{\infty}\left(t^{[\mu]-1-\mu} \sup _{|y|<t}\left\|\partial^{\beta_{1}} u-2 \partial^{\beta_{1}} \tau_{y} u+\partial^{\beta_{1}} \tau_{2 y} u ; L^{r_{1}}\right\|\right)^{2} d t / t\right\}^{1 / 2},
$$

we note that $p \vee k-1+\nu \ell=0$ occurs if and only if $p=k=1$ and $\ell=0$. By (3.8), we have

$$
\Lambda_{3} \leq C\left\|u ; \dot{B}_{r_{1}}^{\mu \theta_{1}}\right\|
$$

where the constant $C$ is independent of $\ell$ since $\theta_{1}$ is in a compact set in $(0,1]$. We also have the estimates of the form (3.16) for $2 \leq j \leq k$. Applying the convex inequalities such as

$$
\left\|u ; \dot{B}_{r_{j}}^{\mu \theta_{j}}\right\| \leq \begin{cases}\left\|u ; \dot{B}_{\tilde{r}}^{\mu}\right\| & \text { if } p=k=1 \quad \text { and } \ell=0 \\ \left\|u ; \dot{B}_{r_{k}(\ell)}^{0}\right\|^{1-\theta_{j}}\left\|u ; \dot{B}_{r}^{\mu}\right\|^{\theta_{j}} \text { otherwise, }\end{cases}
$$

for $1 \leq j \leq k$, we have

$$
\left\|A_{3} \mid\right\| \leq C \sum_{\ell=0}^{\infty} \frac{\kappa^{\ell}}{\ell !}\left\|u ; L^{r_{k}(\ell)} \cap \dot{B}_{r_{k}(\ell)}^{0}\right\|^{p \vee k-1+\nu \ell}\left\|u ; \dot{B}_{r}^{\mu}\right\|+C \delta(p-1)\left\|u ; \dot{B}_{\tilde{r}}^{\mu}\right\| .
$$

Since $k \leq[\mu]-1$, this is one of the estimates that we have required.

For $A_{4}$, first we note that $A_{4}$ is considered only for $k \geq 2$, so that $p \vee k-$ $1+\nu \ell>0$. Let $\theta_{j}, r_{j}, j=1, \ldots, k$, be given by

$$
\begin{aligned}
\theta_{j} & \equiv\left(\mu-[\mu]+1+2\left|\beta_{j}\right|\right) / 2 \mu \quad \text { for } j=1,2, \\
\theta_{j} & \equiv\left|\beta_{j}\right| / \mu \quad \text { for } \quad 3 \leq j \leq k, \\
1 / r_{j} & \equiv\left(1-\theta_{j}\right) / r_{k}(\ell)+\theta_{j} / r \quad \text { for } \quad 1 \leq j \leq k .
\end{aligned}
$$

Then

$$
1 / \tilde{r}=\left((p-k)_{+}+\nu \ell\right) / r_{k}(\ell)+\sum_{j=1}^{k} 1 / r_{j}
$$

so that we have

$$
\left\|A_{4}\right\| \mid \leq C \sum_{\ell=0}^{\infty} \frac{\kappa^{\ell}}{\ell !}\left\|u ; L^{r_{k}(\ell)}\right\|{ }^{(p-k)_{+}+\nu \ell} \prod_{j=3}^{k}\left\|\partial^{\beta_{j}} u ; L^{r_{j}}\right\| \cdot \Lambda_{4},
$$

where

$$
\Lambda_{4} \equiv\left\{\int_{0}^{\infty}\left(t^{[\mu]-1-\mu} \sup _{|y|<t}\left\|\partial^{\beta_{1}} u-\partial^{\beta_{1}} \tau_{y} u ; L^{r_{1}}\right\|\left\|\partial^{\beta_{2}} u-\partial^{\beta_{2}} \tau_{y} u ; L^{r_{2}}\right\|\right)^{2} d t / t\right\}^{1 / 2} .
$$


By the Hölder inequality and (3.7), we have

$$
\Lambda_{4} \leq C \prod_{j=1}^{2}\left\|u ; \dot{B}_{r_{j}, 4}^{\mu \theta_{j}}\right\| \leq C \prod_{j=1}^{2}\left\|u ; \dot{B}_{r_{j}}^{\mu \theta_{j}}\right\|
$$

where we have used (3.9) for the last inequality, and the constant $C$ is independent of $\ell$ since $\theta_{j}, j=1,2$, are in a compact set in $(0,1)$. We also have the estimates of the form (3.16) for $3 \leq j \leq k$. Applying the convex inequalities such as

$$
\left\|u ; \dot{B}_{r_{j}}^{\mu \theta_{j}}\right\| \leq\left\|u ; \dot{B}_{r_{k}(\ell)}^{0}\right\|^{1-\theta_{j}}\left\|u ; \dot{B}_{r}^{\mu}\right\|^{\theta_{j}}
$$

for $1 \leq j \leq k$, we have

$$
\left\|A_{4}\right\| \mid \leq C \sum_{\ell=0}^{\infty} \frac{\kappa^{\ell}}{\ell !}\left\|u ; L^{r_{k}(\ell)} \cap \dot{B}_{r_{k}(\ell)}^{0}\right\|^{p \vee k-1+\nu \ell}\left\|u ; \dot{B}_{r}^{\mu}\right\| .
$$

Since $k \leq[\mu]-1$, this is the last one of the estimates that we have required.

At the end of the proof, we mention the last statement in the proposition. If we use the embedding

$$
\left\|u ; \dot{B}_{r, m}^{\mu}\right\| \leq C\left\|u ; B_{r, m}^{\mu}\right\|
$$

with $\mu>0,1 \leq r, m \leq \infty$ for (3.3), then we have

$$
\left\|f(u) ; \dot{B}_{\tilde{r}}^{\mu}\right\| \leq C \sum_{\substack{\ell=0 \\ p-1+\nu \ell>0}}^{\infty} \frac{\kappa^{\ell}}{\ell !}\left\|u ; L^{r_{1}(\ell)}\right\|^{p-1+\nu \ell}\left\|u ; B_{r}^{\mu}\right\|+C \delta(p-1)\left\|u ; B_{\tilde{r}}^{\mu}\right\|
$$

for $0<\mu<1$ in addition to (3.3). Since the constant $C$ in (3.17) is independent of $r$ and $m$ for any fixed $\mu>0$, we can replace $\dot{B}_{r(\ell), 2 p}^{\mu / p}, \dot{B}_{r(\ell), 4}^{\mu / 2}$ with $B_{r(\ell), 2 p}^{\mu / p}$, $B_{r(\ell), 4}^{\mu / 2}$ in (3.11), (3.13), respectively. Then using the convex inequality

$$
\begin{aligned}
& \left\|u ; B_{r, m}^{\mu}\right\| \leq\left\|u ; B_{r_{1}, m_{1}}^{\mu_{1}}\right\|^{1-\theta}\left\|u ; B_{r_{2}, m_{2}}^{\mu_{2}}\right\|^{\theta} \\
& \mu=(1-\theta) \mu_{1}+\theta \mu_{2}, 1 / r=(1-\theta) / r_{1}+\theta / r_{2}, 1 / m=(1-\theta) / m_{1}+\theta / m_{2}
\end{aligned}
$$

for $\mu, \mu_{1}, \mu_{2} \in \mathbb{R}, 1 \leq r, m, r_{1}, m_{1}, r_{2}, m_{2} \leq \infty$ and $0 \leq \theta \leq 1$ instead of (3.11) and (3.13), we obtain

$$
\begin{aligned}
& \left\|f(u) ; \dot{B}_{\tilde{r}}^{\mu}\right\| \\
& \leq\left\{\begin{array}{cc}
C \sum_{\ell=0, p-1+\nu \ell>0}^{\infty} \frac{\kappa^{\ell}}{\ell !}\left\|u ; L^{r_{1}(\ell)} \cap B_{r_{1}(\ell)}^{0}\right\|^{p-1+\nu \ell}\left\|u ; B_{r}^{\mu}\right\| \\
+C \delta(p-1)\left\|u ; B_{\tilde{r}}^{\mu}\right\| & \text { if } 1 \leq \mu<2 \text { and } \mu<p, \\
C \sum_{k=1}^{2} \sum_{\ell=0, p \vee k-1+\nu \ell>0}^{\infty} \frac{\kappa^{\ell}}{\ell !}\left\|u ; L^{r_{k}(\ell)} \cap B_{r_{k}(\ell)}^{0}\right\|{ }^{p \vee k-1+\nu \ell}\left\|u ; B_{r}^{\mu}\right\| \\
+C \delta(p-1)\left\|u ; B_{\tilde{r}}^{\mu}\right\| & \text { if } 1 \leq \mu<2 \text { and } \mu \geq p .
\end{array}\right.
\end{aligned}
$$


The same argument is also true for $\mu \geq 2$ applying the embedding $B_{r_{j}}^{\mu \theta_{j}} \hookrightarrow \dot{B}_{r_{j}}^{\mu \theta_{j}}$ to $(3.16)$, where the constant appearing in the embedding is independent of $\ell$ since $\theta_{j}$ is in a compact set in $(0,1]$, so that we can replace $\dot{B}$ with $B$ on the RHS of (3.4). And we also have the same result for (3.6).

\section{§4. Proof of Theorem 1.1}

Let $\sigma$ and $\lambda$ satisfy (2.1) with $\sigma=2 \lambda-2$ in the sequel. In particular we can take $\sigma$ as any number which satisfies $n-1 \leq \sigma \leq n$ and $\sigma>0$. Taking $\sigma=n$ in the following argument, we obtain the proof of Theorem 1.1. Let $n, s, s^{*}, p$ be as in Theorem 1.1. Let $p_{0}$ satisfy

$$
1<p_{0} \leq \begin{cases}(1+4 / \sigma) \wedge p & \text { for } \quad p>1 \\ 1+(2 / \sigma) \wedge 1 \wedge \nu & \text { for } \quad p=1\end{cases}
$$

There exist $s_{0}, \tilde{\rho}$ and an admissible pair $\left(\left(1 / q_{0}, 1 / r_{0}\right),(1 / \tilde{q}, 1 / \tilde{r})\right)$ with $0<s_{0} \leq$ $1 / 2, \tilde{\rho} \leq-s_{0}$ and

$$
\begin{gathered}
0<1 / q_{0} \leq \sigma / 2(\sigma+2) \leq 1 / r_{0}<1 / 2, \\
1 / 2 \leq 1 / \tilde{r} \leq(\sigma+4) / 2(\sigma+2) \leq 1 / \tilde{q} \leq 1, \\
1 / \tilde{r}=p_{0} / r_{0}, \quad 1 / \tilde{q}=\theta+p_{0} / q_{0}, \quad \theta \equiv 1-\left(p_{0}-1\right) \sigma / 4, \\
0=-s_{0}+n \alpha\left(r_{0}\right)-2(n-\lambda) / \sigma q_{0} \\
=1+2(n-\lambda) / \sigma+\tilde{\rho}+n \alpha(\tilde{r})-2(n-\lambda) / \sigma \tilde{q} .
\end{gathered}
$$

Indeed, the above $s_{0}, \tilde{\rho}, q_{0}, r_{0}, \tilde{q}, \tilde{r}$ are given by

$$
\begin{aligned}
& s_{0} \equiv \begin{cases}\frac{(\sigma+2)\left(p_{0}-1\right)}{4 p_{0}} \text { if } p_{0}<p_{*} \equiv 1+2 / \sigma, \\
\frac{1}{2} \text { otherwise, }\end{cases} \\
& \tilde{\rho} \equiv \begin{cases}-1 \quad \text { if } p_{0}<p_{*}, \\
-\frac{3}{2}+\frac{\sigma\left(p_{0}-1\right)}{4} \quad \text { otherwise, }\end{cases} \\
& 1 / q_{0} \equiv \sigma s_{0} /(\sigma+2), \quad 1 / r_{0} \equiv 1 / 2-2 / \sigma q_{0}, \quad 1 / \tilde{r} \equiv p_{0} / r_{0}, \quad 1 / \tilde{q} \equiv \theta+p_{0} / q_{0},
\end{aligned}
$$

where the admissibility follows from Corollary 2.1 . 
For any interval $I \subset \mathbb{R}$ let $X_{s_{0}}^{\mu}(I), \mu=s_{0}, s^{*}, s$, be a function space defined by

$$
X_{s_{0}}^{\mu}(I) \equiv L^{\infty}\left(I ; H^{\mu}\right) \cap L^{q_{0}}\left(I ; B_{r_{0}}^{\mu-s_{0}}\right)
$$

with the norm

$$
\left\|u ; X_{s_{0}}^{\mu}(I)\right\| \equiv\left\|u ; L^{\infty}\left(I ; H^{\mu}\right) \cap L^{q_{0}}\left(I ; B_{r_{0}}^{\mu-s_{0}}\right)\right\| .
$$

Then we show the existence of solutions of (NLKG) in the function space defined by

$$
\begin{aligned}
X\left(I, R_{s_{0}}, R_{n / 2}\right) & \equiv\left\{u ;\left\|u ; X_{s_{0}}^{\mu}(I)\right\| \leq R_{\mu}, \mu=s_{0}, n / 2\right\} \quad \text { if } \quad s=n / 2, \\
X\left(I, R_{s_{0}}, R_{s^{*}}, R_{s}\right) & \equiv\left\{u ;\left\|u ; X_{s_{0}}^{\mu}(I)\right\| \leq R_{\mu}, \mu=s_{0}, s^{*}, s\right\} \quad \text { if } \quad s>n / 2,
\end{aligned}
$$

for some $R_{\mu}>0, \mu=s_{0}, s^{*}, s$, endowed with the metric

$$
d(u, v) \equiv\left\|u-v ; L^{\infty}\left(I ; L^{2}\right) \cap L^{q_{0}}\left(I ; L^{r_{0}}\right)\right\| .
$$

First we consider the proof for $s=n / 2$. For any $\ell \geq 0$ and $k \geq 1$ with $p \vee k-1+\nu \ell>0$ let $1 / r_{k}(\ell) \equiv\left(p_{0}-1\right) /(p \vee k-1+\nu \ell) r_{0}$. Then we have

$$
1 / \tilde{r}=(p \vee k-1+\nu \ell) / r_{k}(\ell)+1 / r_{0} .
$$

Let $f$ satisfy $N\left(n / 2,(n / 2+\tilde{\rho})_{+}, p\right)$. Since $r_{0}, \tilde{r}$ satisfy $0<1 / r_{0}<1 / 2,1 / 2 \leq$ $1 / \tilde{r} \leq 1$ and $r_{k}(\ell)$ satisfies $0<1 / r_{k}(\ell) \leq 1 / r_{0}$, applying Proposition 3.1 to the composite function $f(u)$, for any $\mu$ with $-\tilde{\rho}<\mu \leq n / 2$ we have

$$
\begin{aligned}
\left\|f(u) ; \dot{B}_{\tilde{r}}^{\mu+\tilde{\rho}}\right\| \leq & C \sum_{k=1}^{[\mu+\tilde{\rho}]+1} \sum_{\substack{\ell=0 \\
p \vee k-1+\nu \ell>0}}^{\infty} \frac{\kappa^{\ell}}{\ell !}\left\|u ; L^{r_{k}(\ell)} \cap B_{r_{k}(\ell)}^{0}\right\|^{p \vee k-1+\nu \ell}\left\|u ; B_{r_{0}}^{\mu+\tilde{\rho}}\right\| \\
& +C \delta(p-1)\left\|u ; B_{\tilde{r}}^{\mu+\tilde{\rho}}\right\| .
\end{aligned}
$$

Lemma 4.1 [17, Lemma 2.2]. The following estimates hold.

$$
\begin{aligned}
& \left\|u ; L^{r}\right\| \leq C_{0} r^{1 / 2+\left(r_{0}-2\right) / 2 r}\left\|u ; H^{n / 2}\right\|^{1-r_{0} / r}\left\|u ; L^{r_{0}}\right\|^{r_{0} / r}, \\
& \left\|u ; B_{r}^{0}\right\| \leq C_{0} r^{1 / 2+\left(r_{0}-2\right) / 2 r}\left\|u ; H^{n / 2}\right\|^{1-r_{0} / r}\left\|u ; B_{r_{0}}^{0}\right\|^{r_{0} / r}
\end{aligned}
$$

for any $r_{0}, r$ with $0<1 / r \leq 1 / r_{0} \leq 1$, where the constant $C_{0}$ is independent of $r$, but may be dependent on $r_{0}$. 
By Lemma 4.1, we have

$$
\begin{aligned}
\| u ; L^{r_{k}(\ell)} & \cap B_{r_{k}(\ell)}^{0} \| \\
& \leq C_{0} r_{k}(\ell)^{1 / 2+\left(r_{0}-2\right) / 2 r_{k}(\ell)}\left\|u ; H^{n / 2}\right\|^{1-r_{0} / r_{k}(\ell)}\left\|u ; L^{r_{0}} \cap B_{r_{0}}^{0}\right\|^{r_{0} / r_{k}(\ell)},
\end{aligned}
$$

where the constant $C_{0}$ is independent of $\ell$, so that the RHS of (4.7) is estimated by

$$
\begin{aligned}
& \sum_{k=1}^{[\mu+\tilde{\rho}]+1} \sum_{\substack{\ell=0 \\
p \vee k-1+\nu \ell>0}}^{\infty} a_{k}(\ell)\left\|u ; H^{n / 2}\right\|^{p \vee k-1+\nu \ell-\left(p_{0}-1\right)}\left\|u ; B_{r_{0}}^{0}\right\|^{p_{0}-1}\left\|u ; B_{r_{0}}^{\mu+\tilde{\rho}}\right\| \\
& +C \delta(p-1)\left\|u ; B_{\tilde{r}}^{\mu+\tilde{\rho}}\right\|,
\end{aligned}
$$

where we define $a_{k}(\ell)$ by

$$
a_{k}(\ell) \equiv \frac{\kappa^{\ell}}{\ell !} C_{0}^{p \vee k-1+\nu \ell} r_{k}(\ell)^{(p \vee k-1+\nu \ell) / 2+\left(p_{0}-1\right)\left(r_{0}-2\right) / 2 r_{0}} .
$$

Therefore by the fact $\theta \geq 0$, applying the Hölder inequality in the time variable, we have

(4.8) $\left\|f(u) ; L^{\tilde{q}}\left(I ; \dot{B}_{\tilde{r}}^{\mu+\tilde{\rho}}\right)\right\|$

$$
\begin{aligned}
& \leq \sum_{k=1}^{[\mu+\tilde{\rho}]+1} \sum_{\substack{\ell=0 \\
p \vee k-1+\nu \ell>0}}^{\infty} a_{k}(\ell)\left\|u ; L^{\infty}\left(I ; H^{n / 2}\right)\right\|^{p \vee k-1+\nu \ell-\left(p_{0}-1\right)}|I|^{\theta} \\
& \quad \cdot\left\|u ; L^{q_{0}}\left(I ; B_{r_{0}}^{0}\right)\right\|^{p_{0}-1}\left\|u ; L^{q_{0}}\left(I ; B_{r_{0}}^{\mu+\tilde{\rho}}\right)\right\| \\
& \quad+C \delta(p-1)\left\|u ; L^{\tilde{q}}\left(I ; B_{\tilde{r}}^{\mu+\tilde{\rho}}\right)\right\|
\end{aligned}
$$

for any interval $I \subset \mathbb{R}$. Similarly, by (3.2) and the Hölder inequality in space and time variables we also have

$$
\begin{aligned}
(4.9)\left\|f(u) ; L^{\tilde{q}}\left(I ; L^{\tilde{r}}\right)\right\| \leq & \sum_{\substack{\ell=0 \\
p-1+\nu \ell>0}}^{\infty} a_{1}(\ell)\left\|u ; L^{\infty}\left(I ; H^{n / 2}\right)\right\|^{p-1+\nu \ell-\left(p_{0}-1\right)}|I|^{\theta} \\
& \cdot\left\|u ; L^{q_{0}}\left(I ; L^{r_{0}}\right)\right\|^{p_{0}-1}\left\|u ; L^{q_{0}}\left(I ; L^{r_{0}}\right)\right\| \\
& +C \delta(p-1)\left\|u ; L^{\tilde{q}}\left(I ; L^{\tilde{r}}\right)\right\|
\end{aligned}
$$

Therefore by the embedding $B_{r}^{0} \hookrightarrow L^{r}$ for $2 \leq r<\infty$ and the equivalence 
$B_{p, q}^{s}=L^{p} \cap \dot{B}_{p, q}^{s}$ for $s>0$ and $1 \leq p, q \leq \infty$, for any $\mu$ with $\mu+\tilde{\rho} \geq 0$ we have

$$
\begin{aligned}
& \left\|f(u) ; L^{\tilde{q}}\left(I ; B_{\tilde{r}}^{\mu+\tilde{\rho}}\right)\right\| \\
& \leq \sum_{k=1}^{[\mu+\tilde{\rho}]+1} \sum_{\substack{\ell=0 \\
p \vee k-1+\nu \ell>0}}^{\infty} a_{k}(\ell)\left\|u ; L^{\infty}\left(I ; H^{n / 2}\right)\right\|^{p \vee k-1+\nu \ell-\left(p_{0}-1\right)}|I|^{\theta} \\
& \quad \cdot\left\|u ; L^{q_{0}}\left(I ; B_{r_{0}}^{0}\right)\right\|^{p_{0}-1}\left\|u ; L^{q_{0}}\left(I ; B_{r_{0}}^{\mu-s_{0}}\right)\right\| \\
& \quad+C \delta(p-1)|I|\left\|u ; L^{\infty}\left(I ; B_{\tilde{r}}^{\mu}\right)\right\|
\end{aligned}
$$

where we have used the fact that $\tilde{q}=1, \tilde{r}=2$ for $p=1$, and the embeddings $H^{\mu} \hookrightarrow B_{2}^{\mu+\tilde{\rho}}, B_{r_{0}}^{\mu-s_{0}} \hookrightarrow B_{r_{0}}^{\mu+\tilde{\rho}}$. Therefore by (4.9) with the embedding $L^{\tilde{r}} \hookrightarrow$ $B_{\tilde{r}}^{\mu+\tilde{\rho}}$ for $\mu=s_{0}$ or $\mu=n / 2$ with $\mu+\tilde{\rho}<0$, and by (4.10) for $\mu=n / 2$ with $\mu+\tilde{\rho} \geq 0$, we have

$$
\left\|f(u) ; L^{\tilde{q}}\left(I ; B_{\tilde{r}}^{\mu+\tilde{\rho}}\right)\right\| \leq\left(F\left(R_{n / 2}\right)|I|^{\theta} R_{s_{0}}^{p_{0}-1}+C \delta(p-1)|I|\right) R_{\mu}
$$

for any $\mu=s_{0}, n / 2$, and any $u \in X\left(I, R_{s_{0}}, R_{n / 2}\right)$, where $F(\cdot)$ is a nonnegative series defined by

$$
F(\rho) \equiv \sum_{k=1}^{[\mu+\tilde{\rho}]+1} \sum_{\substack{\ell=0 \\ p \vee k-1+\nu \ell>0}}^{\infty} a_{k}(\ell) \rho^{p \vee k-1+\nu \ell-\left(p_{0}-1\right)} .
$$

Here we note that $F(\rho)$ exists for any $\rho \in[0, \infty)$ if $\nu<2$, for any $\rho \in[0, C(\kappa))$ if $\nu=2$, where $C(\kappa)$ is a positive constant behaving as $O\left(\kappa^{-1 / 2}\right)$ both $\kappa \rightarrow 0$ and $\kappa \rightarrow \infty$. This follows from the ratio test with

$$
\lim _{\ell \rightarrow \infty} \frac{a_{k}(\ell+1) \rho^{p \vee k-1+\nu(\ell+1)-\left(p_{0}-1\right)}}{a_{k}(\ell) \rho^{p \vee k-1+\nu \ell-\left(p_{0}-1\right)}}= \begin{cases}0 & \text { if } \quad \nu<2, \\ 2 C_{0}^{2} e \kappa r_{0} \rho^{2} /\left(p_{0}-1\right) & \text { if } \quad \nu=2 .\end{cases}
$$

Now, for any $t_{0} \in \bar{I}$ and any data $(\phi, \psi) \in A^{n / 2}\left(\gamma_{s_{0}}, \gamma_{n / 2}\right)$, let $\Phi_{t_{0}}$ be an operator defined as

$$
\Phi_{t_{0}}(u)(t) \equiv \dot{K}(t) \phi+K(t) \psi+\int_{t_{0}}^{t} K(t-s) f(u(s)) d s .
$$

By the admissibility of $\left(\left(1 / q_{0}, 1 / r_{0}\right),(1 / \tilde{q}, 1 / \tilde{r})\right)$, we have

$$
\begin{aligned}
\left\|\Phi_{t_{0}}(u) ; X_{s_{0}}^{\mu}(I)\right\| & \leq C\left\|(\phi, \psi) ; \mathcal{H}^{\mu}\right\|+C\left\|f(u) ; L^{\tilde{q}}\left(I ; B_{\tilde{r}}^{\mu+\tilde{\rho}}\right)\right\| \\
& \leq C \gamma_{\mu}+\left(F\left(R_{n / 2}\right)|I|^{\theta} R_{s_{0}}^{p_{0}-1}+C \delta(p-1)|I|\right) R_{\mu}
\end{aligned}
$$

for $\mu=s_{0}, n / 2$, any $(\phi, \psi) \in A^{n / 2}\left(\gamma_{s_{0}}, \gamma_{n / 2}\right)$ and any $u \in X\left(I, R_{s_{0}}, R_{n / 2}\right)$. 
On the other hand, by the same argument as above, we also have

$$
d\left(\Phi_{t_{0}}(u), \Phi_{t_{0}}(v)\right) \leq\left(F\left(R_{n / 2}\right)|I|^{\theta} R_{s_{0}}^{p_{0}-1}+C \delta(p-1)|I|\right) d(u, v)
$$

for any $u, v \in X\left(I, R_{s_{0}}, R_{n / 2}\right)$. Indeed, by the embeddings $H^{s_{0}} \hookrightarrow L^{2}$ and $B_{r_{0}}^{0} \hookrightarrow L^{r_{0}}$, we have

$$
\begin{aligned}
& d\left(\Phi_{t_{0}}(u), \Phi_{t_{0}}(v)\right) \\
& \quad \leq C\left\|\int_{t_{0}}^{t} K(t-s)(f(u(s))-f(v(s))) d s ; L^{\infty}\left(I ; H^{s_{0}}\right) \cap L^{q_{0}}\left(I ; B_{r_{0}}^{0}\right)\right\| .
\end{aligned}
$$

Applying Corollary 2.1 to the RHS of the last inequality, we have

$$
d\left(\Phi_{t_{0}}(u), \Phi_{t_{0}}(v)\right) \leq C\left\|f(u)-f(v) ; L^{\tilde{q}}\left(I ; B_{\tilde{r}}^{s_{0}+\tilde{\rho}}\right)\right\| .
$$

By the embedding $L^{\tilde{r}} \hookrightarrow B_{\tilde{r}}^{s_{0}+\tilde{\rho}}$, we obtain

$$
d\left(\Phi_{t_{0}}(u), \Phi_{t_{0}}(v)\right) \leq C\left\|f(u)-f(v) ; L^{\tilde{q}}\left(I ; L^{\tilde{r}}\right)\right\| .
$$

By the Hölder inequality in space and time variables with (4.3) we have

$$
\begin{aligned}
\| f(u)- & f(v) ; L^{\tilde{q}}\left(I ; L^{\tilde{r}}\right) \| \\
\leq & F\left(\left\|u ; L^{\infty}\left(I ; H^{n / 2}\right)\right\| \vee\left\|v ; L^{\infty}\left(I ; H^{n / 2}\right)\right\|\right)|I|^{\theta} \\
& \cdot\left(\left\|u ; L^{q_{0}}\left(I ; L^{r_{0}}\right)\right\| \vee\left\|v ; L^{q_{0}}\left(I ; L^{r_{0}}\right)\right\|\right)^{p_{0}-1}\left\|u-v ; L^{q_{0}}\left(I ; L^{r_{0}}\right)\right\| \\
& +C \delta(p-1)|I|\left\|u-v ; L^{\infty}\left(I ; L^{2}\right)\right\| .
\end{aligned}
$$

Therefore we obtain (4.14).

By the above argument, if $\gamma_{\mu}>0, R_{\mu}>0$ and $I \subset \mathbb{R}$ satisfy

$$
\begin{aligned}
& \delta(\nu-2) 2 C_{0}^{2} e \kappa r_{0} R_{n / 2}^{2}<p_{0}-1, \\
& C \gamma_{\mu}+\left(F\left(R_{n / 2}\right)|I|^{\theta} R_{s_{0}}^{p_{0}-1}+C \delta(p-1)|I|\right) R_{\mu} \leq R_{\mu}, \\
& F\left(R_{n / 2}\right)|I|^{\theta} R_{s_{0}}^{p_{0}-1}+C \delta(p-1)|I| \leq 1 / 2
\end{aligned}
$$

for $\mu=s_{0}, n / 2$, where (4.17) which is from (4.12) ensures the existence of $F\left(R_{n / 2}\right)$ and is disregarded for $\nu \neq 2$, then $\Phi_{t_{0}}$ is a contraction map on $X\left(I, R_{s_{0}}, R_{n / 2}\right)$. Since $X\left(I, R_{s_{0}}, R_{n / 2}\right)$ is a complete metric space, $\Phi_{t_{0}}$ has a unique fixed point in $X\left(I, R_{s_{0}}, R_{n / 2}\right)$ and the solution $u$ of (NLKG) with the data $u(0)=\phi, \partial_{t} u(0)=\psi$ is given by the fixed point of $\Phi_{0}$. Let $R_{\mu} \equiv 2 C \gamma_{\mu}$, $\mu=s_{0}, n / 2$. Then (4.17), (4.18) and (4.19) are rewritten as

$$
\delta(\nu-2) \gamma_{n / 2}<C \kappa^{-1 / 2},
$$




$$
G\left(\gamma_{n / 2}\right)|I|^{\theta} \gamma_{s_{0}}^{p_{0}-1}+C \delta(p-1)|I| \leq 1
$$

for a series $G$ and a positive constant $C$ depending on $p_{0}$ but not on $\phi$ and $\psi$, where (4.20) is disregarded for $\nu \neq 2$. We see that the fixed point $u$ satisfies $\left(u, \partial_{t} u\right) \in C_{b}\left(I ; \mathcal{H}^{n / 2}\right)$ by using Corollary 2.1 with (4.11) and the unitarity of the operator $U(t)$.

The following lemma gives the uniqueness of solutions of (NLKG) in $C\left(I ; H^{n / 2}\right)$ with the same data at some point in $I$.

Lemma 4.2. Let $u, v$ be the solutions of (NLKG) in $C\left(I ; H^{n / 2}\right)$ which satisfy

$$
\left(u(t), \partial_{t} u(t)\right)=\left(v(t), \partial_{t} v(t)\right) \quad \text { in } \quad \mathcal{H}^{n / 2}
$$

for some $t=t_{1} \in I$. If $\nu<2$, then (4.22) holds for all $t \in I$. When $\nu=2$, if $\left\|u ; L^{\infty}\left(I ; H^{n / 2}\right)\right\|$ is sufficiently small, then the same conclusion holds.

Proof of Lemma 4.2. Let $u$ and $v$ satisfy (4.22) for some $t=t_{1} \in I$. Then $u$ and $v$ satisfy

$$
w(t)=\Phi(w)(t)=\dot{K}\left(t-t_{1}\right) u\left(t_{1}\right)+K\left(t-t_{1}\right) \partial_{t} u\left(t_{1}\right)+\int_{t_{1}}^{t} K(t-s) f(w(s)) d s
$$

for $w=u, v$, where the last term on the RHS makes sense as a function with values in $L^{2}$ by Corollary 2.1, an estimate similar to (4.9), and the embedding $H^{n / 2} \hookrightarrow L^{r}$ with $2 \leq r<\infty$. Let $J$ be any compact interval with $t_{1} \in J \subset I$ and let

$$
a \equiv\left\|u ; L^{\infty}\left(J ; H^{n / 2}\right)\right\| \vee\left\|v ; L^{\infty}\left(J ; H^{n / 2}\right)\right\| .
$$

For $\varepsilon>0$ let $I_{t_{1}, \varepsilon} \equiv\left[t_{1}-\varepsilon, t_{1}+\varepsilon\right] \cap J$. By the embedding $H^{n / 2} \hookrightarrow L^{r}$ for $2 \leq r<\infty, u$ and $v$ satisfy $u(t), v(t) \in L^{r_{0}}$ for $t \in I_{t_{1}, \varepsilon}$ and moreover $u, v \in C\left(I_{t_{1}, \varepsilon} ; L^{r_{0}}\right)$ and

$$
\left\|w ; L^{q_{0}}\left(I_{t_{1}, \varepsilon} ; L^{r_{0}}\right)\right\| \leq C a(2 \varepsilon)^{1 / q_{0}}
$$

for $w=u, v$. Therefore similarly to (4.15) and (4.16) we have

$$
\begin{aligned}
\| u-v ; L^{\infty}\left(I_{t_{1}, \varepsilon} ; L^{2}\right) \cap & L^{q_{0}}\left(I_{t_{1}, \varepsilon} ; L^{r_{0}}\right) \| \\
\leq & G(a)(2 \varepsilon)^{\theta+\left(p_{0}-1\right) / q_{0}}\left\|u-v ; L^{q_{0}}\left(I_{t_{1}, \varepsilon} ; L^{r_{0}}\right)\right\| \\
& +C \delta(p-1) 2 \varepsilon\left\|u-v ; L^{\infty}\left(I_{t_{1}, \varepsilon} ; L^{2}\right)\right\|,
\end{aligned}
$$

where $\theta+\left(p_{0}-1\right) / q_{0}>0$ since $\theta \geq 0, p_{0} \neq 1$ and $1 / q_{0} \neq 0$. So that if $\nu<2$, then for sufficiently small $\varepsilon>0$ we have $u(t)=v(t)$ in $L^{2} \cap L^{r_{0}}$ for any $t \in I_{t_{1}, \varepsilon}$, 
which means $u=v$ in $C\left(I_{t_{1}, \varepsilon} ; H^{n / 2}\right)$. Repeating this procedure, we have $u=v$ in $C\left(J ; H^{n / 2}\right)$ and therefore in $C\left(I ; H^{n / 2}\right)$. When $\nu=2$, if $\left\|u ; L^{\infty}\left(I ; H^{n / 2}\right)\right\|$ is sufficiently small, then taking $\varepsilon>0$ sufficiently small, $\left\|v ; L^{\infty}\left(I_{t_{1}, \varepsilon} ; H^{n / 2}\right)\right\|$ is also sufficiently small, so that the above argument is valid and we obtain $u=v$ in $C\left(I ; H^{n / 2}\right)$ analogously.

In the following argument let $\gamma_{n / 2}>0$ be any number which satisfies (4.20).

(1) Let $p_{0}<p_{*}, t_{0}=0$ and $I=[-T, T], T>0$, in the above argument. Then $\tilde{\rho}=-1$. Since $\theta>0$ for $p_{0}<1+4 / \sigma$, there exists $T>0$ which satisfies (4.21) with $\gamma_{s_{0}}$ replaced by $\gamma_{n / 2}$. Then for any $(\phi, \psi) \in \mathcal{H}^{n / 2}$ with $\left\|(\phi, \psi) ; \mathcal{H}^{n / 2}\right\| \leq \gamma_{n / 2},(\mathrm{NLKG})$ has a unique solution $u$ in $X\left(I, R_{s_{0}}, R_{n / 2}\right)$ and the solution $u$ satisfies $\left(u, \partial_{t} u\right) \in C\left(I ; \mathcal{H}^{n / 2}\right)$, where we have used the inequality $\left\|\phi ; H^{s_{1}}\right\| \leq\left\|\phi ; H^{s_{2}}\right\|$ for $s_{1} \leq s_{2}$. The solution $u$ is also a unique solution of (NLKG) in $C\left(I ; H^{n / 2}\right)$ by Lemma 4.2 , where we note that when $\nu=2$, taking $\gamma_{n / 2}>0$ sufficiently small, $R_{n / 2}>0$ is also sufficiently small, so that $\left\|u ; L^{\infty}\left(I ; H^{n / 2}\right)\right\|$ is sufficiently small as required in the assumption in Lemma 4.2.

(2) Let $\nu<2$. We consider the case $T^{*}<\infty$. The proof for $T_{*}<\infty$ follows quite similarly. Let $p_{0}<p_{*}$ in the argument before (1). Now let

$$
\gamma \equiv \sup _{0 \leq t<T^{*}}\left\|\left(u(t), \partial_{t} u(t)\right) ; \mathcal{H}^{n / 2}\right\|<\infty .
$$

Let $\varepsilon>0$ be a number which satisfies

$$
G(\gamma) \varepsilon^{\theta} \gamma^{p_{0}-1}+C \delta(p-1)|I| \leq 1,
$$

where $G$ is the same function appearing in (4.21). Let $t_{0}>0$ be a number such that $T^{*} \in\left(t_{0}, t_{0}+\varepsilon\right)$. Then by the same argument as in (1), the operator $\Phi_{t_{0}, u}$ defined as

$$
\Phi_{t_{0}, u}(v)(t) \equiv \dot{K}\left(t-t_{0}\right) u\left(t_{0}\right)+K\left(t-t_{0}\right) \partial_{t} u\left(t_{0}\right)+\int_{t_{0}}^{t} K(t-s) f(v(s)) d s
$$

has a unique fixed point $\tilde{u}_{+}$in $C\left(\left[t_{0}, t_{0}+\varepsilon\right] ; H^{n / 2}\right)$. Let $\tilde{u}(t)=u(t)$ for $t \in$ $\left[0, t_{0}\right), \tilde{u}(t)=\tilde{u}_{+}(t)$ for $t \in\left[t_{0}, t_{0}+\varepsilon\right]$. Then $\tilde{u}$ is a solution of (NLKG) in $C\left(\left[0, t_{0}+\varepsilon\right] ; H^{n / 2}\right)$ with $\tilde{u}(0)=u(0)$ and $\partial_{t} \tilde{u}(0)=\partial_{t} u(0)$. Since $u=\tilde{u}$ in $C\left(\left[0, T^{*}\right) ; H^{n / 2}\right)$ by Lemma $4.2, u$ can be extended as $\tilde{u}$ beyond $T^{*}$ with value in $H^{n / 2}$, which contradicts the definition of $T^{*}$.

(3) Let $t_{0}=0$ and $I=[-T, T], T>0$. If $1<p<1+4 / \sigma$, then we can take $p_{0}$ as $p_{0}=p$. Let $f$ satisfy $N\left(n / 2,(n / 2+\tilde{\rho})_{+}, p\right)$. Since $\theta \neq 0$, we can 
take $T$ in (4.21) as any number with

$$
T \leq\left(\gamma_{s_{0}}^{p-1} G\left(\gamma_{n / 2}\right)\right)^{-4 /(4-\sigma(p-1))} .
$$

So that we conclude (1.2) by (4.23).

(4) If $p$ satisfies $p \geq 1+4 / \sigma$, then we can take $p_{0}$ for $p_{0}=1+4 / \sigma$ and therefore $s_{0}=1 / 2$ and $\theta=0$. Since $G$ in (4.21) is independent of $I$, therefore there exists small $\gamma_{s_{0}}$ such that

$$
G\left(\gamma_{n / 2}\right) \gamma_{s_{0}}^{4 / \sigma}<1
$$

holds corresponding to (4.21).

(4a) With above $\gamma_{s_{0}}>0$ fixed, for any $(\phi, \psi) \in A^{n / 2}\left(\gamma_{s_{0}}, \gamma_{n / 2}\right)$ we see that $\Phi$ is a contraction map on $X\left(\mathbb{R}, R_{s_{0}}, R_{n / 2}\right)$ for some $R_{\mu}>0, \mu=s_{0}, n / 2$. The fixed point $u$ satisfies $\left(u, \partial_{t} u\right) \in C_{b}\left(\mathbb{R} ; \mathcal{H}^{n / 2}\right)$, and is unique in $C\left(\mathbb{R} ; H^{n / 2}\right)$ by Lemma 4.2, where we note that when $\nu=2$, taking $\gamma_{n / 2}>0$ sufficiently small, $R_{n / 2}>0$ is also sufficiently small, so that $\left\|u ; L^{\infty}\left(I ; H^{n / 2}\right)\right\|$ is sufficiently small as required in the assumption in Lemma 4.2.

Let $\left(\phi_{+}, \psi_{+}\right)$and $\left(\phi_{-}, \psi_{-}\right)$be defined by

$$
\phi_{ \pm}=\phi+\int_{0}^{ \pm \infty} K(-s) f(u(s)) d s, \quad \psi_{ \pm}=\psi+\int_{0}^{ \pm \infty} \dot{K}(-s) f(u(s)) d s
$$

Then $\left(\phi_{ \pm}, \psi_{ \pm}\right) \in \mathcal{H}^{n / 2}$. Indeed, by Corollary 2.1, we have

$$
\begin{aligned}
\left\|\phi_{ \pm} ; H^{n / 2}\right\| & \leq\left\|\phi ; H^{n / 2}\right\|+\left\|\int_{t}^{ \pm \infty} K(t-s) f(u(s)) d s ; L^{\infty}\left(\mathbb{R} ; H^{n / 2}\right)\right\| \\
& \leq\left\|\phi ; H^{n / 2}\right\|+C\left\|f(u) ; L^{\tilde{q}}\left(\mathbb{R} ; B_{\tilde{r}}^{n / 2+\tilde{\rho}}\right)\right\|,
\end{aligned}
$$

where we have used the continuity of $K *_{t} f(u)$ with respect to the time variable. Since the last term of the last inequality in (4.26) is estimated as (4.11) with $|I|^{\theta}$ replaced by $1,\left\|\phi_{ \pm} ; H^{n / 2}\right\|$ is finite. It follows that $\psi_{ \pm} \in H^{n / 2-1}$ analogously.

With these $\phi_{ \pm}$and $\psi_{ \pm},(1.3)$ holds. Indeed, since $v_{ \pm}$are rewritten as

$$
v_{ \pm}(t)=\dot{K}(t) \phi+K(t) \psi+\int_{0}^{ \pm \infty} K(t-s) f(u(s)) d s
$$

by the same argument in (4.26) we have

$$
\begin{aligned}
\left\|u(t)-v_{+}(t) ; H^{n / 2}\right\| & \leq\left\|\int_{\tau}^{\infty} K(\tau-s) f(u(s)) d s ; L_{\tau}^{\infty}\left([t, \infty) ; H^{n / 2}\right)\right\| \\
& \leq C\left\|f(u) ; L^{\tilde{q}}\left([t, \infty) ; B_{\tilde{r}}^{n / 2+\tilde{\rho}}\right)\right\| .
\end{aligned}
$$

Since the RHS of the last inequality is estimated by (4.11) with $|I|^{\theta}$ replaced by 1 , and is bounded uniformly in $t$, we conclude that $u(t)-v_{+}(t)$ converges 
to zero in $H^{n / 2}$ as $t \rightarrow \infty$. By an analogous argument for $\partial_{t} u(t)-\partial_{t} v_{+}(t)$, $u(t)-v_{-}(t)$ and $\partial_{t} u(t)-\partial_{t} v_{-}(t)$, we conclude (1.3).

To show the uniqueness of the pairs $\left(\phi_{ \pm}, \psi_{ \pm}\right)$in (1.3), it suffices to show that

$$
\lim _{t \rightarrow \infty}\left\|\left(\dot{K}(t) \phi_{0}+K(t) \psi_{0}, \partial_{t}\left(\dot{K}(t) \phi_{0}+K(t) \psi_{0}\right)\right) ; \mathcal{H}^{n / 2}\right\|=0
$$

implies $\phi_{0}=\psi_{0}=0$. If (4.28) holds, then we have

$$
\lim _{t \rightarrow \infty}\left\|U(t)\left(\phi_{0}-i \omega^{-1} \psi_{0}\right) ; H^{n / 2}\right\| \vee\left\|U(-t)\left(\phi_{0}+i \omega^{-1} \psi_{0}\right) ; H^{n / 2}\right\|=0,
$$

where $\omega \equiv(1-\Delta)^{1 / 2}$. Therefore by the unitarity of $U(t)$, we conclude $\phi_{0}=$ $\psi_{0}=0$.

(4b) For any data $\left(\phi_{-}, \psi_{-}\right) \in A^{n / 2}\left(\gamma_{s_{0}}, \gamma_{n / 2}\right)$ let $\Phi_{-\infty}$ be an operator defined as

$$
\Phi_{-\infty}(u)(t) \equiv \dot{K}(t) \phi_{-}+K(t) \psi_{-}+\int_{-\infty}^{t} K(t-s) f(u(s)) d s .
$$

By the argument before (1) in this section, $\Phi_{-\infty}$ has a unique fixed point $u$ in $X\left(\mathbb{R}, R_{s_{0}}, R_{n / 2}\right)$, where $R_{\mu}>0, \mu=s_{0}, n / 2$, are chosen to satisfy $R_{\mu}=2 C \gamma_{\mu}$, $\mu=s_{0}, n / 2$, with the same constant $C$ appearing in (4.18). Let $(\phi, \psi)$ and $\left(\phi_{+}, \psi_{+}\right)$be defined by the equations $(4.25)$. Then, as in the argument on (4.26), we have $(\phi, \psi),\left(\phi_{+}, \psi_{+}\right) \in \mathcal{H}^{n / 2}$. Now $u$ is rewritten as

$$
u(t)=\dot{K}(t) \phi+K(t) \psi+\int_{0}^{t} K(t-s) f(u(s)) d s,
$$

which implies exactly that $u$ is a solution of (NLKG) in $X\left(\mathbb{R}, R_{s_{0}}, R_{n / 2}\right)$ with the data $u(0)=\phi, \partial_{t} u(0)=\psi . \quad u$ is also a unique solution of (NLKG) in $C\left(\mathbb{R} ; H^{n / 2}\right)$ which satisfies

$$
\left\|\left(u(t)-v_{-}(t), \partial_{t}\left(u(t)-v_{-}(t)\right)\right) ; \mathcal{H}^{n / 2}\right\| \rightarrow 0
$$

as $t \rightarrow-\infty$, where $v_{-}(t) \equiv \dot{K}(t) \phi_{-}+K(t) \psi_{-}$. Indeed, first we show $u$ is a unique solution of (NLKG) in $C\left(\mathbb{R} ; H^{n / 2}\right) \cap X_{s_{0}}^{n / 2}(\mathbb{R})$ which satisfies (4.29). Let $v$ be a solution of (NLKG) in $C\left(\mathbb{R} ; H^{n / 2}\right) \cap X_{s_{0}}^{n / 2}(\mathbb{R})$ which satisfies (4.29) with $u$ replaced by $v$. Then $v$ satisfies $v=\Phi_{-\infty}(v)$, and for sufficiently small $t_{0}<0$ $v$ satisfies

$$
\left\|v ; X_{s_{0}}^{\mu}\left(\left(-\infty, t_{0}\right]\right)\right\| \leq R_{\mu}=2 C \gamma_{\mu}
$$


for any $\mu=s_{0}, n / 2$, since

$$
\lim _{t \rightarrow-\infty}\left|\left\|v(t) ; H^{\mu}\right\|-\left\|v_{-}(t) ; H^{\mu}\right\|\right| \vee\left|\left\|\partial_{t} v(t) ; H^{\mu-1}\right\|-\left\|\partial_{t} v_{-}(t) ; H^{\mu-1}\right\|\right|=0
$$

by (4.29) with $u$ replaced by $v$, and $\left\|\left(v_{-}(t), \partial_{t} v_{-}(t)\right) ; \mathcal{H}^{\mu}\right\| \leq \gamma_{\mu}$ by the unitarity of the free propagator, namely

$$
\left\|\left(v_{-}(t), \partial_{t} v_{-}(t)\right) ; \mathcal{H}^{\mu}\right\|=\left\|\left(\phi_{-}, \psi_{-}\right) ; \mathcal{H}^{\mu}\right\|
$$

for any $t \in \mathbb{R}$. Therefore $v$ is also a fixed point of $\Phi_{-\infty}$ in

$$
X\left(\left(-\infty, t_{0}\right], R_{s_{0}}, R_{n / 2}\right) .
$$

Since $\Phi_{-\infty}$ has a unique fixed point in $X\left(\left(-\infty, t_{0}\right], R_{s_{0}}, R_{n / 2}\right)$, we have $u(t)=$ $v(t)$ for any $t \in\left(-\infty, t_{0}\right]$. Especially we have $u\left(t_{0}\right)=v\left(t_{0}\right)$ and $\partial_{t} u\left(t_{0}\right)=$ $\partial_{t} v\left(t_{0}\right)$. Therefore we conclude $u=v$ in $C\left(\mathbb{R} ; H^{n / 2}\right)$ by Lemma 4.2. Second we show $u$ is a unique solution of (NLKG) in $C\left(\mathbb{R} ; H^{n / 2}\right)$ which satisfies (4.29). Let $v$ be a solution of (NLKG) in $C\left(\mathbb{R} ; H^{n / 2}\right)$ which satisfies (4.29) with $u$ replaced by $v$. Then $v$ satisfies

$$
\limsup _{t \rightarrow-\infty}\left\|\left(v(t), \partial_{t} v(t)\right) ; \mathcal{H}^{\mu}\right\| \leq \gamma_{\mu}
$$

for any $\mu=s_{0}, n / 2$, by (4.30) and (4.31). Therefore there exists sufficiently small $t_{0}<0$ such that

$$
G\left(\left\|\left(v\left(t_{0}\right), \partial_{t} v\left(t_{0}\right)\right) ; \mathcal{H}^{n / 2}\right\|\right)\left\|\left(v\left(t_{0}\right), \partial_{t} v\left(t_{0}\right)\right) ; \mathcal{H}^{s_{0}}\right\|^{4 / \sigma}<1
$$

corresponding to (4.24). Now let $\Phi_{t_{0}, v}$ be an operator defined by

$$
\Phi_{t_{0}, v}(w) \equiv \dot{K}\left(t-t_{0}\right) v\left(t_{0}\right)+K\left(t-t_{0}\right) \partial_{t} v\left(t_{0}\right)+\int_{t_{0}}^{t} K(t-s) f(w(s)) d s .
$$

Then by the condition (4.32) and the same argument in (4a), $\Phi_{t_{0}, v}$ has a unique fixed point in $X\left(\mathbb{R}, R_{s_{0}}^{\prime}, R_{n / 2}^{\prime}\right)$ for some $R_{\mu}^{\prime}>0, \mu=s_{0}, n / 2$, and the fixed point is in $C\left(\mathbb{R} ; H^{n / 2}\right)$. Since $v$ is a fixed point of $\Phi_{t_{0}, v}$ with $v \in C\left(\mathbb{R} ; H^{n / 2}\right)$, by the uniqueness of solutions of (NLKG) in $C\left(\mathbb{R} ; H^{n / 2}\right) v$ must satisfy

$$
v \in X\left(\mathbb{R}, R_{s_{0}}^{\prime}, R_{n / 2}^{\prime}\right) \subset X_{s_{0}}^{n / 2}(\mathbb{R}) .
$$

Therefore by the uniqueness in $C\left(\mathbb{R} ; H^{n / 2}\right) \cap X_{s_{0}}^{n / 2}(\mathbb{R})$, we conclude $u=v$. So that $u$ is a unique solution of (NLKG) in $C\left(\mathbb{R} ; H^{n / 2}\right)$ which satisfies (4.29). 
Similarly to the argument on (4.27) we have (1.3) for these $u,\left(\phi_{+}, \psi_{+}\right)$, $\left(\phi_{-}, \psi_{-}\right)$, and we also have the uniqueness of $\left(\phi_{+}, \psi_{+}\right)$for $\left(\phi_{-}, \psi_{-}\right)$with (1.3).

By the above argument, we are able to define the scattering operator $S$ on $A^{n / 2}\left(\gamma_{s_{0}}, \gamma_{n / 2}\right)$ to $\mathcal{H}^{n / 2}$ as $S\left(\left(\phi_{-}, \psi_{-}\right)\right)=\left(\phi_{+}, \psi_{+}\right)$by way of a unique global solution $u$ of $(\mathrm{NLKG})$ in $C\left(\mathbb{R} ; H^{n / 2}\right)$ with (1.3). Moreover, the correspondence is given by (4.25) or equivalently by

$$
\phi_{+}=\phi_{-}+\int_{-\infty}^{\infty} K(-s) f(u(s)) d s, \quad \psi_{+}=\psi_{-}+\int_{-\infty}^{\infty} \dot{K}(-s) f(u(s)) d s .
$$

We show the continuity of the operator $S$. For any

$$
\left\{\left(\phi_{-}^{j}, \psi_{-}^{j}\right)\right\}_{j=1}^{\infty} \subset A^{n / 2}\left(\gamma_{s_{0}}, \gamma_{n / 2}\right)
$$

which satisfies (1.4), let $\left\{u^{j}\right\}_{j=1}^{\infty}$ be the fixed points in $X\left(\mathbb{R}, R_{s_{0}}, R_{n / 2}\right)$ of the operators $\left\{\Phi_{-\infty}^{j}\right\}_{j=1}^{\infty}$ given by

$$
\Phi_{-\infty}^{j}(v)(t) \equiv \dot{K}(t) \phi_{-}^{j}+K(t) \psi_{-}^{j}+\int_{-\infty}^{t} K(t-s) f(v(s)) d s
$$

for $j \geq 1$. Then by Corollary 2.1 we have, as in (4.14),

$d\left(\Phi_{-\infty}^{j}(u), \Phi_{-\infty}^{j}\left(u^{j}\right)\right) \leq C\left\|\left(\phi_{-}-\phi_{-}^{j}, \psi_{-}-\psi_{-}^{j}\right) ; \mathcal{H}^{s_{0}}\right\|+F\left(R_{n / 2}\right) R_{s_{0}}^{p_{0}-1} d\left(u, u^{j}\right)$

for any $j \geq 1$. Since (4.19) holds with $|I|^{\theta}$ replaced by 1 , we conclude that $d\left(u, u^{j}\right) \rightarrow 0$ as $j \rightarrow \infty$. On the other hand, by (4.33), we have

$$
\begin{aligned}
\left\|\phi_{+}-\phi_{+}^{j} ; H^{s_{0}}\right\| \leq & \left\|\phi_{-}-\phi_{-}^{j} ; H^{s_{0}}\right\| \\
& +\left\|\int_{-\infty}^{\infty} K(t-s)\left(f(u(s))-f\left(u^{j}(s)\right)\right) d s ; L^{\infty}\left(\mathbb{R} ; H^{s_{0}}\right)\right\|
\end{aligned}
$$

for any $j \geq 1$. By Corollary 2.1, the same argument used on (4.16) shows that the last term on the RHS of the last inequality is estimated by

$$
F\left(R_{n / 2}\right) R_{s_{0}}^{p_{0}-1} d\left(u, u^{j}\right) .
$$

Therefore we have $\left\|\phi_{+}-\phi_{+}^{j} ; H^{s_{0}}\right\| \rightarrow 0$ as $j \rightarrow \infty$. By the embedding $H^{s_{0}} \hookrightarrow$ $H^{\nu}$ for $\nu \leq s_{0}$, we also have $\left\|\phi_{+}-\phi_{+}^{j} ; H^{\nu}\right\| \rightarrow 0$ as $j \rightarrow \infty$ for any $\nu$ with $\nu \leq s_{0}$. By the convex inequality

$$
\begin{aligned}
\| \phi_{+} & -\phi_{+}^{j} ; H^{\nu} \| \\
& \leq\left\|\phi_{+}-\phi_{+}^{j} ; H^{s_{0}}\right\|^{(n / 2-\nu) /\left(n / 2-s_{0}\right)}\left\|\phi_{+}-\phi_{+}^{j} ; H^{n / 2}\right\|^{\left(\nu-s_{0}\right) /\left(n / 2-s_{0}\right)},
\end{aligned}
$$


we have $\left\|\phi_{+}-\phi_{+}^{j} ; H^{\nu}\right\| \rightarrow 0$ as $j \rightarrow \infty$ for any $\nu$ with $s_{0} \leq \nu<n / 2$. Analogously we also have $\left\|\psi_{+}-\psi_{+}^{j} ; H^{\nu-1}\right\| \rightarrow 0$ as $j \rightarrow \infty$ for any $\nu$ with $\nu<n / 2$. Therefore we obtain (1.5).

(5) Let $t_{0}=0$ and let $I=[-T, T]$ for (1), $I=\mathbb{R}$ for (4a). Since $\left\{u_{j}\right\}_{j=1}^{\infty}$ satisfies

$$
u_{j}(t)=\dot{K}(t) \phi_{j}+K(t) \psi_{j}+\int_{0}^{t} K(t-s) f\left(u_{j}(s)\right) d s
$$

for any $j \geq 1$, by Corollary 2.1 and the embedding $L^{\tilde{r}} \hookrightarrow B_{\tilde{r}}^{0}$ for $1<\tilde{r} \leq 2$, we have

$$
d\left(u, u_{j}\right) \leq C\left\|\left(\phi-\phi_{j}, \psi-\psi_{j}\right) ; \mathcal{H}^{s_{0}}\right\|+C\left\|f(u)-f\left(u_{j}\right) ; L^{\tilde{q}}\left(I ; L^{\tilde{r}}\right)\right\|
$$

for any $j \geq 1$, where the constant $C$ is independent of $I,(\phi, \psi), u,\left\{\left(\phi_{j}, \psi_{j}\right)\right\}_{j=1}^{\infty}$ and $\left\{u_{j}\right\}_{j=1}^{\infty}$. As we have shown in (1) and (4a), for any given initial data in $A^{n / 2}\left(\gamma_{s_{0}}, \gamma_{n / 2}\right)$ the unique solution of (NLKG) in $C\left(\mathbb{R} ; H^{n / 2}\right)$ is given in the restricted space $X\left(I, R_{s_{0}}, R_{n / 2}\right)$, where $R_{\mu}>0, \mu=s_{0}, n / 2$, are chosen to satisfy $R_{\mu}=2 C \gamma_{\mu}, \mu=s_{0}, n / 2$, and $C$ is the same constant appearing in (4.18). Therefore $u,\left\{u_{j}\right\}_{j=1}^{\infty}$ are in $X\left(I, R_{s_{0}}, R_{n / 2}\right)$. So that by the same argument on (4.16), we have

$$
C\left\|f(u)-f\left(u_{j}\right) ; L^{\tilde{q}}\left(I ; L^{\tilde{r}}\right)\right\| \leq\left(F\left(R_{n / 2}\right)|I|^{\theta} R_{s_{0}}^{p_{0}-1}+C \delta(p-1)|I|\right) d\left(u, u_{j}\right),
$$

where $|I|^{\theta}$ and $C \delta(p-1)|I|$ are disregarded for (4a). Since (4.19) holds for these $R_{\mu}, \mu=s_{0}, n / 2$, we conclude that $d\left(u, u_{j}\right) \rightarrow 0$ as $j \rightarrow \infty$. Especially we obtain

$$
\left\|u-u_{j} ; L^{\infty}\left(I ; H^{s_{0}}\right)\right\| \rightarrow 0 \quad \text { as } j \rightarrow \infty,
$$

from which $\left\|u-u_{j} ; L^{\infty}\left(I ; H^{\nu}\right)\right\| \rightarrow 0$ as $j \rightarrow \infty$ for $\nu<n / 2$ follows by the embedding and the convex inequality as in the last part in (4b) in this section. Concerning

$$
\left\|\partial_{t}\left(u-u_{j}\right) ; L^{\infty}\left(I ; H^{\nu-1}\right)\right\| \rightarrow 0
$$

as $j \rightarrow \infty$ for any $\nu$ with $\nu<n / 2$, the proof is analogous and omitted. Consequently we obtain the required results.

For $s>n / 2$, the sufficient conditions to show that $\Phi_{t_{0}}$ is a contraction map on $X\left(I, R_{s_{0}}, R_{s^{*}}, R_{s}\right)$ are given by

$$
C \gamma_{\mu}+M\left(R_{s^{*}}\right)|I|^{\kappa} R_{s_{0}}^{p_{0}-1} R_{\mu} \leq R_{\mu}, \quad M\left(R_{s^{*}}\right)|I|^{\kappa} R_{s_{0}}^{p_{0}-1} \leq 1 / 2
$$


for $\mu=s_{0}, s^{*}, s$, which could be rewritten as $M\left(\gamma_{s^{*}}\right)|I|^{\theta} \gamma_{s_{0}}^{p_{0}-1} \leq 1$ independently of $\gamma_{s}$. The remaining proof for $s>n / 2$ is carried out along the lines of the proof for $s=n / 2$.

\section{References}

[1] Bergh, J. and Löfström, J., Interpolation Spaces, Grundlehren Math. Wiss., SpringerVerlag, Berlin-New York, 1976.

[2] Brenner, P., On space-time means and strong global solutions of nonlinear hyperbolic equations, Math. Z., 201 (1989), 45-55.

[3],$- L_{p}$-estimates of difference schemes for strictly hyperbolic systems with nonsmooth data, SIAM J. Numer. Anal., 14 (1977), 1126-1144.

[4] Cazenave, T. and Weissler, F. B., The Cauchy problem for the critical nonlinear Schrödinger equation in $H^{s}$, Nonlinear Anal., 14 (1990), 807-836.

[5] Ginibre, J. and Velo, G., Generalized Strichartz inequalities for the wave equation, J. Funct. Anal., 133 (1995), 50-68.

[6] - The global Cauchy problem for the nonlinear Schrödinger equation revisited, Ann. Inst. H. Poincaré Anal. Non Linéaire, 2 (1985), 309-327.

[7] - The global Cauchy problem for the nonlinear Klein-Gordon equation, Math. Z., 189 (1985), 487-505.

[8] Kato, T., On nonlinear Schrödinger equations, II. $H^{s}$-solutions and unconditional wellposedness, J. Anal. Math., 67 (1995), 281-306.

[9] Lindblad, H., A sharp counterexample to the local existence of low-regularity solutions to nonlinear wave equations, Duke Math. J., 72 (1993), 503-539.

[10] Lindblad, H. and Sogge, C. D., On existence and scattering with minimal regularity for semilinear wave equations, J. Funct. Anal., 130 (1995), 357-426.

[11] Marshall, B., Strauss, W. and Wainger, S., $L^{p}-L^{q}$ estimates for the Klein-Gordon equation, J. Math. Pures Appl., 59 (1980), 417-440.

[12] Motai, T., Existence of global strong solution for nonlinear Klein-Gordon equation, Funkcial. Ekvac., 32 (1989), 125-144.

[13] Nakamura, M. and Ozawa, T., Small solutions to nonlinear wave equations in the Sobolev spaces, to appear in Houston J. Math.

[14] - Small solutions to nonlinear Schrödinger equations in the Sobolev spaces, $J$. D'Anal. Math., 81 (2000), 305-329.

[15] - Global solutions in the critical Sobolev space for the wave equations with nonlinearity of exponential growth, Math. Z., 231 (1999), 479-487.

[16] - The Cauchy problem for nonlinear wave equations in the homogeneous Sobolev space, Ann. Inst. H. Poincaré, Phys. Théor., 71 (1999), 199-215.

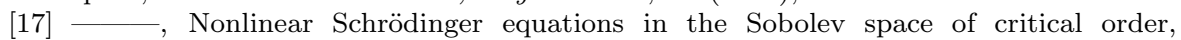
J. Funct. Anal., 150 (1998), 364-380.

[18] - Low energy scattering for nonlinear Schrödinger equations in fractional order Sobolev spaces, Rev. Math. Phys., 9 (1997), 397-410.

[19] Nakanishi, K., Scattering theory for the nonlinear Klein-Gordon equation with Sobolev critical power, Internat. Math. Res. Notices, No.1 (1999), 31-60.

[20] Pecher, H., Low energy scattering for nonlinear Klein-Gordon equations, J. Funct. Anal., 63 (1985), 101-122.

[21] - Nonlinear small data scattering for the wave and Klein-Gordon equation, Math. Z., 185 (1984), 261-270.

[22] Rauch, J., I. The $u^{5}$ Klein-Gordon equation, II. Anomalous singularities for semilinear wave equations, Nonlinear partial differential equations and their applications, Collège de France Seminar, Vol. I (Paris, 1978/1979), pp. 335-364, Res. Notes Math., 53, Pitman, Boston, Mass.-London, (1981). 
[23] Strauss, W., Nonlinear scattering theory at low energy, J. Funct. Anal., 41 (1981), 110-133.

[24] Strichartz, R. S., Restrictions of Fourier transforms to quadratic surfaces and decay of solutions of wave equations, Duke Math. J., 44 (1977), 705-714.

[25] - A note on Trudinger's extension of Sobolev's inequalities, Indiana Univ. Math. J., 21 (1972), 841-842.

[26] Triebel, H., Theory of Function Spaces, Birkhäuser, Basel, 1983.

[27] Tsutsumi, M. and Hayashi, N., Scattering of solutions of nonlinear Klein-Gordon equations in higher space dimensions, Recent topics in nonlinear PDE, (Hiroshima, 1983), 221-239, North-Holland Math. Stud., 98, North-Holland, Amsterdam-New York, 1984.

[28] Tsutsumi, M., Scattering of solutions of nonlinear Klein-Gordon equations in three space dimensions, J. Math. Soc. Japan, 35 (1983), 521-538.

[29] Wang, B., Scattering of solutions for critical and subcritical nonlinear Klein-Gordon equations in $H^{s}$, Discrete Contin. Dynam. Systems, 5 (1999), 753-763.

[30] - On existence and scattering for critical and subcritical nonlinear Klein-Gordon equations in $H^{s}$, Nonlinear Anal., 31 (1998), 573-587. 